\title{
Nutrition and Exercise Strategies to Prevent Excessive Pregnancy Weight Gain: A Meta-analysis
} \author{
Kathleen M. Antony, MD, MSCI ${ }^{5}$ Cynthia K. Wautlet, MD, MPH \\ ${ }^{1}$ Department of Integrated Biology, University of Wisconsin - \\ Madison, Madison, Wisconsin \\ ${ }^{2}$ Department of Biostatistics and Medical Informatics, University of \\ Wisconsin - Madison, Madison, Wisconsin \\ ${ }^{3}$ Division of Infectious Disease, Department of Medicine, University of \\ Wisconsin-Madison School of Medicine and Public Health, Madison, \\ Wisconsin \\ ${ }^{4}$ Department of Medicine, William S. Middleton Memorial Veterans \\ Hospital, Madison, Wisconsin \\ ${ }^{5}$ Department of Obstetrics and Gynecology, University of Wisconsin - \\ Madison, Madison, Wisconsin
}

Katherine A. Craemer, BS ${ }^{1,5}$ Emmanuel Sampene, $\mathrm{PhD}^{2} \quad$ Nasia Safdar, MD, PhD ${ }^{3,4}$

Am J Perinatol Rep 2019;9:e92-e120.
Address for correspondence Cynthia K. Wautlet, MD, MPH, University of Wisconsin-Madison, McConnell Hall 4th floor, 1010 Mound Street, Madison, WI 53715 (e-mail: cwautlet@wisc.edu).

\author{
Abstract \\ Keywords \\ - nutrition intervention \\ - exercise intervention \\ - pregnancy \\ - weight gain \\ - obesity
}

Objective To evaluate nutrition-only, exercise-only, and nutrition-plus-exercise interventions for optimizing gestational weight gain (GWG) based on the 2009 Institute of Medicine (IOM) guidelines.

Study PubMed, Google Scholar, and 2015 Cochrane Review were searched. Analysis of variance was used to determine if significant GWG differences exist between strategies, with additional subanalyses on overweight (OV) or obese women based on 2009 IOM guidelines. Results Of 66 identified studies, 31 contributed data $(n=8,558)$. Compared with routine prenatal care, nutrition-only interventions were significantly associated with reduced GWG and are most likely to produce weight gain within IOM recommendations $(p=0.013)$. Exercise-only $(p=0.069)$ and nutrition-plus-exercise $(p=0.056)$ interventions trended toward GWG within IOM guidelines, but did not reach statistical significance. Supervised $(p=0.61)$ and unsupervised $(p=0.494)$ exercise programs had similar effectiveness. Subanalyses on OV or obese women produced similar results to studies that did not differentiate results based on body mass index: nutrition only ( $p=0.011)$, exercise only $(p=0.308)$, and nutrition plus exercise $(p=0.129)$.

Conclusion Preventing excessive GWG is crucial, especially for OV or obese women. In the current study, nutrition-based intervention is the health system strategy that showed significant impact on preventing excessive GWG compared with routine prenatal care. Among women who are OV or have obesity, nutrition-only interventions hold the most promise compared with routine prenatal care.
More women are entering pregnancy as overweight (OV) or having obesity $(\mathrm{OB})$ than in the past, and many are gaining excessive weight during pregnancy. During 2011 to 2014, $34.4 \%$ of the U.S. women of childbearing age (aged 20-39 years) are OV or have OB. ${ }^{1}$ For women who gave birth in 2016,
$21 \%$ aged 18 to 24 years, $23 \%$ aged 25 to 34 years, and $24 \%$ aged 35 to 44 years had $O$ OB. $^{2}$ Weight gain exceeding the Institute of Medicine (IOM) recommendations, known as excessive gestational weight gain (GWG), increases the burden of chronic disease and can put the mother and her infant's health at risk. received

June 16, 2018

accepted after revision

December 11, 2018
DOI https://doi.org/

10.1055/s-0039-1683377. ISSN 2157-6998.
Copyright $\odot 2019$ by Thieme Medical Publishers, Inc., 333 Seventh Avenue, New York, NY 10001, USA. Tel: +1(212) 584-4662.
License terms

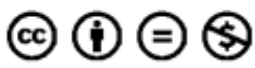


These burdens include, but are not limited to, gestational hypertension, preeclampsia, gestational diabetes, cesarean section delivery, and preterm birth. ${ }^{2}$ Additionally, children of mothers who gain more weight during pregnancy are at higher risk of being OV in early childhood. ${ }^{3}$

Based on the 2009 IOM guidelines, the recommended amount of GWG for underweight women (body mass index [BMI] $\left.<18.5 \mathrm{~kg} / \mathrm{m}^{2}\right)$ is 12.5 to $18 \mathrm{~kg}(28-40 \mathrm{lb})$, normal weight (NW) women (BMI $18.5-24.9 \mathrm{~kg} / \mathrm{m}^{2}$ ) is 11.5 to $16 \mathrm{~kg}(25-35 \mathrm{lb})$, OV women (BMI $\left.25.0-29.9 \mathrm{~kg} / \mathrm{m}^{2}\right)$ is 7 to $11.5 \mathrm{~kg}(15-25 \mathrm{lb})$, and women who have $\mathrm{OB}\left(\mathrm{BMI}>30.0 \mathrm{~kg} / \mathrm{m}^{2}\right)$ is 5 to $9 \mathrm{~kg}(11-20 \mathrm{lb}){ }^{4}$ Previous studies have addressed the ability to control GWG and prevent weight exceeding IOM recommendations where all forms of interventions were considered statistically significant: nutrition only, ${ }^{5-8}$ nutrition only for OV/OB women, ${ }^{9-12}$ nutrition plus exercise, ${ }^{5,13-21}$ nutrition plus exercise for $\mathrm{OV} / \mathrm{OB}$ women, ${ }^{10,22-26}$ exercise only, ${ }^{17}$ and exercise only for $\mathrm{OV} / \mathrm{OB}$ women. ${ }^{10,27-29}$ The study expands on the 2015 Cochrane Review (Muktabhant et al [2015]) assessment of methods involving nutrition, exercise, and combination of nutritionplus-exercise intervention studies to prevent excessive pregnancy weight gain based on studies published after the 2009 IOM recommendations. The Cochrane Review found that whether women participated in nutrition, exercise, or both interventions, their risk of excessive GWG was reduced by an average of $20 \%$ and women of intervention groups were more likely to experience low GWG than those in control groups. ${ }^{30}$ The Cochrane Review and other previous reviews included studies prior to 2009, which could not have used the updated IOM guidelines. A review of interventions that only includes studies published after 2009 is necessary. This meta-analysis exclusively uses studies published after updated IOM guidelines were available. The objective of this study is to quantitatively assess the effect of three health system strategies on GWG: nutrition-only, exercise-only, and combination of nutritionplus-exercise interventions.

\section{Methods}

PubMed and Google Scholar databases were searched weekly from September 20, 2016, through October 29, 2016. All studies included in 2015 Cochrane Review were examined.

Initial key terms search produced 5,528,591 results. Key terms include "pregnancy," "body mass index (BMI)," "nutrition," “exercise," “counseling," “obesity," “overweight," or "intervention." Limiting publish dates from 2009 to 2016 produced 1,199,520 results. Studies published before 2009 were assumed to not have used the 2009 IOM recommendations for GWG, and therefore, ineligible for this meta-analysis. "Gestational weight gain" filters narrowed results to 14,827 . All studies were assessed: 12,363 excluded based on irrelevant title; 2,398 excluded based on irrelevant abstract. Full articles for the remaining 66 studies were obtained. Preferred Reporting Items for Systematic Reviews and Meta-Analyses (PRISMA) framework was used for search and reporting of studies. Search was performed by primary author (K.A.C.). Not all aspects of PRISMA were addressed, including risk of bias across studies, due to single person data collection.
Reasons for exclusion include: mean GWG not reported, study published before 2009, and study in trial state ( - Fig. 1). Inclusion criteria: randomized controlled parallel or crosssectional study; at least 20 singleton pregnant women; women aged 18 years or older; control group with standard obstetric care; report means of GWG based on baseline BMI or pre-pregnancy BMI; and use 2009 IOM guidelines for GWG. For studies that did not use the 2009 IOM guidelines, additional analysis was made based on the reported mean GWG.

Data were collected for total GWG in kilogram based on the BMI $\left(\mathrm{kg} / \mathrm{m}^{2}\right)$ of women prior to pregnancy, age at the beginning of gestation, and pre-pregnancy BMI. Statistical analysis was done using Excel Version 2016 (Microsoft, Santa Rosa, CA, United States) and online Vassar Stats application ${ }^{\mathrm{a}}$. Due to strong evidence of heterogeneity between studies, using the random effect approach, we addressed the source of this heterogeneity using subgroup analyses. Analysis of variance (ANOVA) was performed using Vassar Stats to compare effect of intervention and control groups within each study and relative effectiveness of health system strategies. The summary measure in this meta-analysis was the standardized mean difference, defined as the ratio of the difference in mean outcome between the groups and the standard deviation of the outcome among participants. Additional analysis on supervised versus unsupervised exercise was completed. Furthermore, we provided the average GWG means for interventions and subgroups, mean differences, and 95\% confidence intervals in the results section.

\section{Results}

\section{Study Search}

Using the search terms, 66 results were identified. Thirty-five were excluded for not meeting criteria. Thirty-one involving 8,558 participants met criteria and contributed data to these analyses. Of these 31 studies, 6 were nutrition only, ${ }^{31-36} 22$ were nutrition plus exercise,,$^{37-55}$ and 11 were exercise only. ${ }^{51,56-61}$ Four were used twice because data of participants with NW, OV, and having OB were analyzed separately. ${ }^{48,59-61}$ Two were used twice because they reported data of different interventions within studies. ${ }^{51,52}$ One included interventions based on exercise only and nutrition plus exercise. ${ }^{51}$ One included interventions based on low intensity (LI) and moderate intensity (MI) exercise. ${ }^{52}$ Results for all interventions reported separately were treated as separate studies. After separation of data, the 31 studies are analyzed as 39 studies.

\section{Participants}

These 39 studies involved 8,558 pregnant participants. Each study included 23 to 1,108 participants. No statistically significant differences in maternal age were reported. All studies required participants to be $>18$ years old and have singleton pregnancies. Studies recruited up to 26 weeks of gestation. Two recruited at the first prenatal visit. ${ }^{36,61}$ One recruited at the 12 th week of clinic. ${ }^{47}$ One recruited at 6 to

\footnotetext{
a One-way ANOVA. 2010. Vassarstars.net. [accessed October 9, 2016, to June 30, 2017]. http://vassarstats.net/anova1u.html
} 


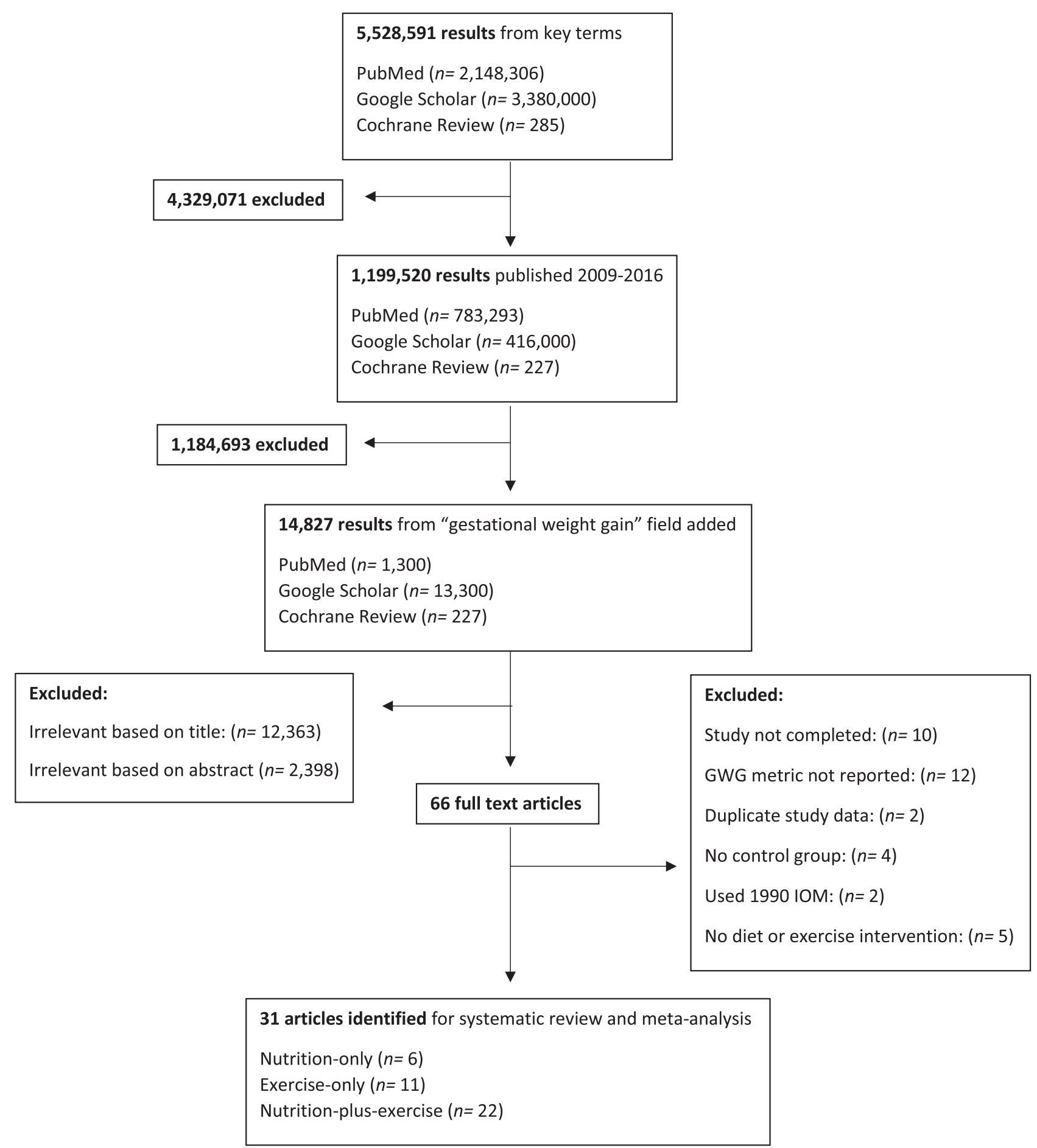

Fig. 1 Electronic search strategy and inclusion criteria for studies used in meta-analysis.

16 weeks. ${ }^{38}$ One required 7 to 21 weeks. ${ }^{55}$ One recruited at eight to nine weeks. ${ }^{46}$ Two required 8 to 12 weeks. ${ }^{44,45}$ One required 10 to 14 weeks. ${ }^{54}$ One required 10 to 16 weeks. ${ }^{48}$ One required 10 to 18 weeks. $^{34}$ One required 10 to 20 weeks. $^{53}$ Two required $<12$ weeks. ${ }^{39,56}$ One required $<13$ weeks. ${ }^{33}$ One required $<14$ weeks. ${ }^{37}$ One required 14 to 24 weeks. ${ }^{60}$ Two required $<15$ weeks. ${ }^{40,59}$ One required 15 to 18 weeks. ${ }^{49}$ Two required $<16$ weeks. ${ }^{41,51}$ One required 16 to 20 weeks. ${ }^{52}$ Two required $<18$ weeks. ${ }^{50,57}$ One required $<20$ weeks. $^{43}$ One required $<24$ weeks. ${ }^{58}$ One required $<26$ weeks. ${ }^{42}$ Three studies did not report the gestational age requirement for participants. $31,32,35$

Weight categories included NW (BMI $18.5-24.9 \mathrm{~kg} / \mathrm{m}^{2}$ ), OV (BMI 25.0-29.9 $\mathrm{kg} / \mathrm{m}^{2}$ ), and women with OB (BMI >30.0). Seventeen studies selected from general population without BMI specifications. 5,32-34,36-40,42,43,46,48,50,52,54,58 Three selected for OV participants. ${ }^{31,47,53}$ Three selected for participants who are OV or have OB. ${ }^{35,44,59}$ Eight selected for participants who have OB. ${ }^{40,45,49,51,55-57,60}$ No significant differences in pre-pregnancy BMI. 
Additional inclusion criteria include: nulliparous participants $^{32,37}$; secundigravida women previously given birth to macrosomic newborn ${ }^{32}$; women expecting second pregnancy ${ }^{36}$; healthy Caucasian mothers ${ }^{54}$; no structured exercise program ( $>60$ minutes once per week) 6 months before trial $^{58}$; sedentary (exercising for $<20$ minutes on $<3$ days/ week) before study ${ }^{61}$; not have engaged $<3,30$-minute exercise per week for 6 months preceding enrollment, ${ }^{60}$ nondiabetic ${ }^{42}$; have at least one of the following risk factors: BMI $>25 \mathrm{~kg} / \mathrm{m}^{2}$; gestational diabetes mellitus or any signs of glucose intolerance or newborn's macrosomia $(>4,500 \mathrm{~g})$ in early pregnancy; type 1 or 2 diabetes in first- or seconddegree relatives; aged $>40$ years. ${ }^{45}$

\section{Setting}

Based on World Bank classifications from 2017 economy, 28 studies occurred in high-income countries. ${ }^{31,32,34-36,40-59,61}$ Three studies occurred in upper middle-income countries. ${ }^{33,39,60}$ No studies occurred in low-income countries.

Eleven studies specified treatment locations: university hospitals $^{33,47,51,54,57,59}$; regular hospitals $32,55,56$; eight multiethnic hospitals ${ }^{49}$; obstetric clini $^{38,60}$; and six primary care maternity health clinics. ${ }^{46}$ All other studies did not specify treatment locations.

\section{Intervention Implementation}

Of the six nutrition-only studies, three focused on low glycemic index (LGI) foods. ${ }^{32,34,36}$ All studies used a food diary to document past eating habits for nutrition plans and to detail food consumption during the trial. Three provided individual nutrition plans. ${ }^{31-33}$ One provided focused nutritional advice based on the macronutrient composition of the participant's diet. ${ }^{33}$ Three provided participants with lists of healthy foods based on local affordability ${ }^{35}$ or LGI foods. ${ }^{34,36}$ Three provided education in group settings. ${ }^{34-36}$ General advice on nutrition, such as a pamphlet, is standard prenatal care and was not considered a nutrition-only intervention.

Of the seven exercise-only studies, four were supervised and three were unsupervised. Two studies included three supervised sessions per week, ${ }^{57,60}$ one required participants to attend at least two sessions per week, ${ }^{58}$ and one had one session per week. ${ }^{60}$ Three supervised studies also had unsupervised exercise to be completed outside of the supervised sessions. ${ }^{56,58,60}$ Types of exercise differed based on the study. One advised exercise based on an expenditure goal of $900 \mathrm{kcal} / \mathrm{wk}$ by means of a walking protocol that took place in five stages of $\mathrm{VO} 2$ measured for oxygen cost. ${ }^{56} \mathrm{~A}$ heart rate monitor was provided to track exercise. One was based on a dance class and core exercises. ${ }^{58}$ Two included aerobic and strength exercises. ${ }^{57,60}$ One also included stretching. ${ }^{60}$ One included aerobic, resistance, and core exercises. ${ }^{61}$ One utilized pedometers ${ }^{51}$ and one provided treadmills. ${ }^{59}$ One registered daily steps on 7 consecutive days every 4 weeks and reminded participants of the recording period starting via text message. ${ }^{51}$

Of the 19 nutrition-plus-exercise studies, 5 included group sessions and 14 provided one-on-one advice. Thirteen were unsupervised and six were supervised. Of the unsupervised interventions, three included DVD instructional videos for home exercise. ${ }^{42,43,49}$ Additional implementations included: weight goal setting by the mother, ${ }^{43,49,53}$ extrasupport for individuals not within IOM recommendations in which exercise and nutritional recommendations were revised, ${ }^{38,39,48}$ food $\log ,{ }^{39,40,43,48,50}$ pedometers, ${ }^{48,51}$ text messages to remind participants when the daily steps recording period of 7 consecutive days every 4 weeks started, ${ }^{51}$ profile-II nutritional program with six subscales to measure health behaviors, ${ }^{39}$ exercises on reading food labels and shopping methods, ${ }^{40}$ used Food Choice Map software, ${ }^{42,43}$ free fitness membership, ${ }^{54}$ heart rate monitors, ${ }^{52}$ and calories calculated based on Dietary Approaches to Stop Hypertension (DASH) dietary pattern and reduced by $30 \%$ for participants without $\mathrm{OB} .{ }^{55}$ In addition to meeting the 2009 IOM recommendations, one study had the goal to keep weight within $3 \%$ of their weight at randomization. ${ }^{55}$

-Fig. 2 provides an overview of the study design and characteristics of included studies.

\section{Effects of Interventions}

Among the total obstetric population studied, results of studies published after the 2009 IOM guidelines indicate that the nutrition-only intervention produced significant GWG differences between mean intervention and mean control groups $(p=0.013)$. Nutrition-plus-exercise $(p=0.056)$ and exercise-only $(p=0.069)$ interventions trended toward statistical significance and show potential to control GWG (-Tables 1 and 2). ANOVA comparison of GWG between all intervention and control groups produced significant results ( $p=0.001$ ) ( - Table $\mathbf{1}$ ).

Graphically, there is a visually noticeable difference between mean control and mean intervention GWG for all intervention groups; however, nutrition $(p=0.013)$ is the only statistically significant health system strategy (-Fig. $\mathbf{3}$ ). Exercise and nutrition plus exercise demonstrated some improvement; however, neither was statistically significant. When separated into supervised $(p=0.61)$ and unsupervised $(p=0.494)$ exercise programs, results were not significant.

-Table 3 depicts upper and lower 95\% confidence intervals for each study based on population $(N)$ and standard deviation (SD). - Fig. 4 depicts the forest plot. Not all studies reported number of participants at, above, or below IOM recommendations, so odds ratio, risk ratio, nor weight could not be calculated.

Eight studies categorized based on pre-pregnancy BMI and analyzed whether mean GWG, as adjusted for BMI, fell below, within, or above IOM recommendations. Three of 8 (37.5\%) exercise-only, 3 of 6 (50\%) nutrition-only, and 15 of 22 (68.18\%) nutrition-plus-exercise interventions were within the IOM GWG recommendations. Compared with control groups within IOM, 3 of 8 (37.5\%) exercise-only, 1 of 8 (12.5\%) nutrition-only, and 6 of $22(27.27 \%)$ nutrition-plus-exercise interventions produced GWG within IOM recommendation. For one of the nutrition-plus-exercise studies, the control GWG fell below the IOM recommendation. ${ }^{37}$

Of the studies that reported percentage of participants exceeding IOM, 2 of 2 (100\%) nutrition only, 9 of 13 (69.23\%) nutrition plus exercise, and 4 of 6 (66.67\%) exercise only 
reported that women in the intervention group exceeded the IOM recommendations less than control group. One exercise-only study on mothers with $O B$ reported the same percentage exceeding IOM for intervention and control groups $^{59}$ (- Table 4).

\section{Participants Who Are Overweight and/or Have Obesity}

Additional analysis was completed on studies that selected for participants who are OV or have OB (-Table 2). When examined individually, 2 of 2 nutrition only (100\%), 7 of 10

Althuizen et al. 2013

\begin{tabular}{ll}
\hline Methods & RCT, February 2005-May 2006 \\
& Location: Netherlands \\
\hline \multirow{3}{*}{ Participants } & 219 randomized participants \\
Inclusion criteria: expecting first child; able to read, write and speak \\
Dutch; within 14 weeks gestation \\
Exclusion criteria: NR
\end{tabular}

Asbee et al. 2009

\begin{tabular}{|c|c|}
\hline Methods & $\begin{array}{l}\text { RCT, Oct 2005-April } 2007 \\
\text { Location: Resident Obstetrics Clinic, Charlotte, North Carolina }\end{array}$ \\
\hline Participants & $\begin{array}{l}100 \text { randomized participants } \\
\text { Inclusion criteria: 6-16 weeks gestation; 18-49 years, prenatal care at } \\
\text { Resident Obstetrics Clinic; English-speaking, Spanish-speaking, or both; } \\
\text { singleton pregnancy } \\
\text { Exclusion criteria: >16 weeks gestation; non-English or non-Spanish- } \\
\text { speaking; multiple pregnancy; BMI >40; preexisting diabetes; untreated } \\
\text { thyroid disease, or hypertension requiring medication; medical } \\
\text { conditions that might affect body weight; delivery at institution other } \\
\text { than Carolinas Medical Center-Main; premature delivery (<37 weeks); } \\
\text { limited prenatal care (<4 visits) }\end{array}$ \\
\hline Intervention & $\begin{array}{l}\text { Intervention: ( } n=57 \text { ) Recommendations for a patient-focused caloric } \\
\text { value divided into } 40 \% \text { carbohydrate, } 30 \% \text { protein, and } 30 \% \text { fat fashion. } \\
\text { Instructed to engage in moderate-intensity exercise } \geq 3 \text { times per week } \\
\text { and preferably } 5 \text { times per week. Received information on appropriate } \\
\text { weight gain during pregnancy using the IOM guidelines. Each participant } \\
\text { met with the dietician only at the time of enrollment. If weight gain not } \\
\text { within the IOM guidelines, participant's diet and exercise regimen was } \\
\text { reviewed and changed. } \\
\text { Control: }(n=43)\end{array}$ \\
\hline Results & $\begin{array}{l}\text { Intervention group GWG: } 13.0 \pm 5.7 \mathrm{~kg} \\
\text { Control group GWG: } 16.2 \pm 7.0 \mathrm{~kg} \\
\text { p-value: }<0.01\end{array}$ \\
\hline
\end{tabular}

Fig. 2 Characteristics of included studies. 
Aşcl et al. 2016

\begin{tabular}{ll}
\hline Methods & RCT \\
& Location: Istanbul, Turkey \\
\hline & 102 randomized participants \\
Inclusion criteria: $\leq 12$ weeks gestation; $\geq 18$ years; gravidity $\leq 2 ;$ no health \\
problems; did not intend to lose weight in pre-pregnancy; got pregnant \\
in natural ways for 2 times at most \\
Exclusion criteria: NR \\
Intervention: ( $n=45)$ nutritional data on a 3-day log and healthy lifestyle \\
behaviors measured using Profile-II with 6 subscales. Four meetings on \\
healthy lifestyle, nutrition, exercise, and weight follow-up, given weight \\
card. Women reaching their objectives were praised and encouraged. \\
Women not meeting objectives were reviewed and regimens intensified. \\
Low-level aerobic exercises recommended for pregnancy were shown \\
and performed, recommended to do mild-moderate safe exercise types \\
to increase heart rate to maximum 140 beats/min for 30 min every \\
other day. \\
Control: (n=45) \\
Intervention \\
$\begin{array}{l}\text { Intervention group GWG: } 12.5 \pm 5.0 \text { kg } \\
\text { Control group GWG: } 12.3 \pm 4.8 \text { kg } \\
\text { p-value: } 0.001\end{array}$ \\
\hline
\end{tabular}

Bogaerts et al. 2012

\begin{tabular}{ll}
\hline Methods & RCT \\
& Location: Flanders, Belgium \\
\hline & 205 randomized participants \\
Inclusion criteria: BMI $>29 ;$ singleton \\
Exclusion criteria: moved out of region; $>15$ weeks gestation; pre- \\
existing type 1 diabetes; multiple pregnancy, primary need for \\
nutritional advice, insufficient knowledge of the Dutch language
\end{tabular}

Fig. 2 (Continued) 
Intervention group $1 \mathrm{GWG}$ : $9.5 \pm 6.8 \mathrm{~kg}$

Intervention group $2 \mathrm{GWG}: 10.6 \pm 7.0 \mathrm{~kg}$

Results

Control group GWG: $13.5 \pm 7.3 \mathrm{~kg}$

p-value I1 vs C: 0.04

p-value I1 vs C: 0.008

Byrne et al. 2011

\begin{tabular}{|c|c|}
\hline Methods & $\begin{array}{l}\text { RCT } \\
\text { Location: Royal Brisbane and Women's Hospital, Queensland, Australia }\end{array}$ \\
\hline Participants & $\begin{array}{l}50 \text { randomized participants } \\
\text { Inclusion criteria: } 18-45 \text { years; BMI } \geq 30 \text {; pregnancy care at the Royal } \\
\text { Brisbane and Women's Hospital; willing and able to do exercise } \\
\text { intervention; able to provide informed consent } \\
\text { Exclusion criteria: non-English speaking; contradiction or inability to } \\
\text { exercise; medical or obstetric contraindication to exercise; multiple } \\
\text { gestation; severe anemia, chronic bronchitis; type } 1 \text { diabetes, } \\
\text { orthopedic limitations; poorly controlled seizure disorder; poorly } \\
\text { controlled hyperthyroidism; or heavy smoker }\end{array}$ \\
\hline Intervention & $\begin{array}{l}\text { Intervention: ( } n=12 \text { ) whole group information session on general advice } \\
\text { on exercise, diet based on the Australian Guide to Healthy Eating and } \\
\text { weight gain based on IOM recommendations. Individual one-on-one } \\
\text { session with midwife. Advised exercise energy expenditure goal of } 900 \\
\text { kcal/week via walking protocol in } 5 \text { stages of speed with VO2 measure } \\
\text { for oxygen cost. Provided with heart rate monitor to track work. } \\
\text { Control: }(n=11)\end{array}$ \\
\hline Results & $\begin{array}{l}\text { Intervention group GWG: } 10.8 \pm 5.1 \mathrm{~kg} \\
\text { Control group GWG: } 11.8 \pm 5.9 \mathrm{~kg} \\
\text { p-value: NR (stated as insignificant) }\end{array}$ \\
\hline \multicolumn{2}{|c|}{ Di Carlo et al. 2014} \\
\hline Methods & $\begin{array}{l}\text { Retrospective, controlled study, January 2010-January } 2011 \\
\text { Location: Italy }\end{array}$ \\
\hline Participants & $\begin{array}{l}154 \text { randomized participants } \\
\text { Inclusion criteria: NR } \\
\text { Exclusion criteria: significant maternal condition (excluding hypertension } \\
\text { and thyroid diseases); multiple pregnancy; } \mathrm{BMI} \leq 20 \text { and } \geq 40 \text { gestational } \\
\text { diabetes; miscarriage or preterm delivery }\end{array}$ \\
\hline Intervention & $\begin{array}{l}\text { Intervention: }(n=77) \text { interviewed about diet, given personalized diet } \\
\text { plan based on food frequency questionnaire (FFQ) } \\
\text { Control: }(n=77)\end{array}$ \\
\hline
\end{tabular}

Fig. 2 (Continued) 
Intervention group GWG: $8.2 \pm 4.0 \mathrm{~kg}$

Results

Control group GWG: $13.4 \pm 4.2 \mathrm{~kg}$

p-value: $<0.001$

Garnæs et al. 2016

$\begin{array}{ll}\text { Methods } & \text { RCT } \\ \text { Location: Norwegian University of Science and Technology (NTNU) and } \\ \text { St. Olavs Hospital, Trondheim University Hospital, Trondheim, Norway }\end{array}$

\begin{tabular}{ll}
\hline & 91 randomized participants \\
& Inclusion criteria: $\mathrm{BMI} \geq 28 \mathrm{~kg} / \mathrm{m} 2, \geq 18$ years, $<18$ gestation, singleton \\
& live fetus at $11-14$ week ultrasound scan; able to come to St. Olavs \\
& Hospital for assessments and exercise classes \\
Exclusion criteria: high risk for preterm labor; diseases that could \\
interfere with participation; habitual exercise training (twice or more \\
weekly) before inclusion
\end{tabular}

Haaksted et al. 2011

\begin{tabular}{ll}
\hline Methods & Parallel study \\
& Location: Oslo, Norway \\
\hline & 105 randomized participants \\
Inclusion criteria: Nulliparous; pre-pregnancy exercise levels did not \\
include participation in structured exercise program ( $>60$ min once per \\
week), with brisk walking ( $>120$ min per week) for six months; able to \\
read, understand and speak Norwegian; $<24$ weeks gestation \\
Exclusion criteria: history of $>2$ miscarriages; severe heart disease \\
(including symptoms of angina, myocardial infarction or arrhythmias); \\
persistent bleeding after 12 weeks gestation; multiple pregnancy; poorly \\
controlled thyroid disease; pregnancy-induced hypertension or pre- \\
eclampsia; unable to attend weekly exercise classes
\end{tabular}

Fig. 2 (Continued) 
e100 Nutrition and Exercise Strategies to Prevent Excessive GWG Craemer et al.

Intervention: ( $n=52$ ) supervised sessions of aerobic dance exercises for 60 minutes with 35 minutes of dance and 15 minutes of core exercises,

Intervention at least 2 times per week, for a minimum of 12 weeks. Asked to exercise 30 minutes/day outside of classes.

Control: $(n=5)$

Intervention group GWG: $13.0 \pm 4.0 \mathrm{~kg}$

Results

Control group GWG: $13.8 \mathrm{~kg} \pm 4.0$

p-value: 0.31

Horan et al. 2016

\begin{tabular}{|c|c|}
\hline Methods & $\begin{array}{l}\text { RCT } \\
\text { Location: National Maternity Hospital, Ireland }\end{array}$ \\
\hline Participants & $\begin{array}{l}800 \text { randomized participants } \\
\text { Inclusion criteria: secundigravida women previously given birth to } \\
\text { macrosomic baby; sufficient literacy and English language fluency to } \\
\text { understand the intervention and can complete questionnaires; healthy; } \\
\text { singleton; no intrauterine growth abnormalities } \\
\text { Exclusion criteria: NR }\end{array}$ \\
\hline Intervention & $\begin{array}{l}\text { Intervention: }(n=138) \text { received low glycemic index }(G I) \text { dietary advice } \\
\text { based on 3-day food diary per trimester. } \\
\text { Control: }(n=142)\end{array}$ \\
\hline Results & $\begin{array}{l}\text { Intervention group GWG: } 13.3 \pm 4.48 \mathrm{~kg} \\
\text { Control group GWG: } 13.7 \pm 4.93 \mathrm{~kg} \\
\text { p-value: } 0.52\end{array}$ \\
\hline
\end{tabular}

Huang et al. 2011

\begin{tabular}{ll}
\hline Methods & $\begin{array}{l}\text { 3-arm RCT, January-June } 2006 \\
\text { Location: Norther Taiwan }\end{array}$ \\
\hline & 189 randomized participants \\
Inclusion criteria: $\geq 18$ years; no cognitive impairment or psychiatric \\
illness; ability to speak and read Chinese; not participating in another \\
study; intention to give birth at the study site \\
Exclusion criteria: NR
\end{tabular}

Fig. 2 (Continued) 
Intervention: ( $n=61$ ) personalized diet and exercise plan with 6 one-toone counselling sessions of one primary session (about 30-40 minutes) at 16-week gestation visit, and five one-to-one booster sessions ( 28

Intervention gestational weeks, 36-38 gestational weeks, before hospital discharge after three-seven-day stay, six weeks post-partum and three months post-partum). Given chart of weight changes after each visit.

Control: $(n=64)$

Intervention group GWG: $14.0 \pm$
Control group GWG: $16.2 \pm 3.3 \mathrm{k}$
$\mathrm{p}$-value: $<0.001$

p-value: $<0.001$

Hui et al. 2011

\begin{tabular}{|c|c|}
\hline Methods & $\begin{array}{l}\text { RCT } \\
\text { Location: Winnipeg }\end{array}$ \\
\hline Participants & $\begin{array}{l}190 \text { randomized participants } \\
\text { Inclusion criteria: nondiabetic; <26 weeks gestation; living in Winnipeg } \\
\text { Exclusion criteria: medical or obstetric contradictions to exercise; having } \\
\text { diabetes }\end{array}$ \\
\hline Intervention & $\begin{array}{l}\text { Intervention: }(n=102) \text { community-based group exercise sessions. } \\
\text { Instructed exercise or mild-to-moderate exercise for } 30-45 \\
\text { minutes/session at 3-5 times/week. Provided } 1 \text { group session/week and } \\
\text { the rest at home with video. Dietary counselling } 2 \text { times using Food } \\
\text { Choice Map. } \\
\text { Control: }(n=88)\end{array}$ \\
\hline Results & $\begin{array}{l}\text { Intervention group GWG: } 14.1 \pm 6.0 \mathrm{~kg} \\
\text { Control group GWG: } 15.2 \pm 5.9 \mathrm{~kg} \\
\text { p-value: } 0.28\end{array}$ \\
\hline
\end{tabular}

Hui et al. 2014

\begin{tabular}{ll}
\hline Methods & RCT, May 2009-December 2011 \\
& Location: Winnipeg, Manitoba \\
\hline & 57 randomized participants \\
Inclusion criteria: $<20$ weeks gestation; no existing diabetes during \\
pregnancy; signed consent form \\
Exclusion criteria: $<3$ times at group exercise; showed no interest to \\
exercise at home; no record of exercise in logbook
\end{tabular}

Fig. 2 (Continued) 
e102 Nutrition and Exercise Strategies to Prevent Excessive GWG Craemer et al.

Intervention: $(n=30)$ weekly community exercise program or unsupervised DVD at home. Mild-to-moderate aerobic exercise, stretching, and strength, encouraged to exercise for 3-5 times a week, 30-45 minutes/time. One-on-one private dietary consultation at

Intervention baseline and at 2 months after using a Food Choice Map (FCM) software. Sticker board food log with portion sizes and frequency. Nutritional recommendation based on calorie intake and macronutrient analysis. Weight goal.

Control: $(n=27)$

Intervention group GWG: $12.9 \pm 3.7 \mathrm{~kg}$

Results Control group GWG: $16.2 \pm 4.4 \mathrm{~kg}$

p-value: $<0.05$

Kong et al. 2014

\begin{tabular}{|c|c|}
\hline Methods & $\begin{array}{l}\text { RCT } \\
\text { Location: lowa State University, Des Moines, lowa }\end{array}$ \\
\hline Participants & $\begin{array}{l}37 \text { randomized participants } \\
\text { Inclusion criteria: age } 18-45 \text { years; singleton; nonsmoker; self-reported } \\
\mathrm{BMI} 26.0-29.9 \mathrm{~kg} / \mathrm{m} 2 \text { or } \geq 30.0 \mathrm{~kg} / \mathrm{m} 2 \text {; no history of chronic diseases; no } \\
\text { history of gestational diabetes; engaged in less than 3, 30-min bouts of } \\
\text { leisure physical activity for } 6 \text { months preceding enrollment } \\
\text { Exclusion criteria: NR }\end{array}$ \\
\hline Intervention & $\begin{array}{l}\text { Intervention: ( } n=9 \text { overweight; } n=9 \text { obese) unsupervised walking } \\
\text { program. Verbally given } 2009 \text { U.S. physical activity guidelines. Provided } \\
\text { treadmills and asked to walk for } 50 \text { min (week } 1 \text { ), } 100 \text { minutes (week } 2 \text { ), } \\
30 \text { min most days of the week (week } 3 \text { to end) for an overall total of at } \\
\text { least } 150 \text { minutes of weekly moderate physical activity } \\
\text { Control: ( } n=9 \text { overweight; } n=10 \text { obese) }\end{array}$ \\
\hline Results & $\begin{array}{l}\text { Intervention groups GWG: } \\
\text { OV: } 10.5 \pm 5.4 \mathrm{~kg} \\
\text { OB: } 12.1 \pm 9.0 \mathrm{~kg} \\
\text { Control groups GWG: } \\
\text { OV: } 9.9 \pm 6.1 \mathrm{~kg} \\
\text { OB: } 12.5 \pm 8.5 \mathrm{~kg} \\
\text { p-value: } 0.859\end{array}$ \\
\hline
\end{tabular}

Korpi-Hyövälti et al. 2011

Methods

RCT, April 2005- May 2006

Location: Finland

Fig. 2 (Continued) 
54 randomized participants Inclusion criteria: $\geq 1$ risk factors: $\mathrm{BMI}>25$ $\mathrm{kg} / \mathrm{m} 2$, previous history of GDM or birth of child $>4.5 \mathrm{~kg}$, age $>40$ years, Participants family history of diabetes, or the venous plasma glucose concentration after 12 hours fasting in the morning was $4.8-5.5 \mathrm{mmol} / \mathrm{I}$ and 2-hour OGTT plasma glucose $<7.8 \mathrm{mmol} / \mathrm{l}$

Exclusion criteria: NR

Intervention: $(n=27)$ women educated on how to eat based on Diabetes and Nutrition Study Group (DNSG) of European Association for the Study of Diabetes. Energy intake suggested $30 \mathrm{kcal} / \mathrm{kg} /$ day for normal weight women and $25 \mathrm{kcal} / \mathrm{kg} /$ day for overweight women. Given specific

Intervention dietary advice 6 times by physiotherapist. Goal of exercise intervention was 30 minutes of daily physical activity for woman who previously exercised $<2.5$ hours per week, and 45 minutes if the woman already engaged in 2.5 hours or more per week of physical activity.

Control: $(n=27)$

Intervention group GWG: $11.4 \mathrm{~kg} \pm 6.0$

Results Control group GWG: $13.9 \mathrm{~kg} \pm 5.1$ p-value: 0.062

Luo et al. 2014

Cross-sectional study, June 2010-Dec 2011

Location: Obstetrics and Gynecology Department of the Second Affiliate

Methods Hospital of the ChongQing University of Medical Sciences, Chongqing, China

\section{6 randomized participants}

Participants Inclusion criteria: <13 weeks gestation; non-smoking; $>18$ years Exclusion criteria: previous history of GDM or other concomitant disease

Intervention: ( $n=131)$ individualized nutrition plan regarding the

Intervention recommended macronutrient composition of their diet.

Control: $(n=145)$

Intervention group GWG: $7.6 \pm 1.6 \mathrm{~kg}$

Results Control group GWG: $12.6 \pm 4.6 \mathrm{~kg}$

p-value: $<0.001$

Luoto et al. 2011

Methods

Cluster RCT

Location: Finland

Fig. 2 (Continued) 


\section{9 randomized participants}

Inclusion criteria: $\geq 1$ risk factors: body mass index (BMI) $>25 \mathrm{~kg} / \mathrm{m} 2$ based on measured height and self-reported pre-pregnancy weight; GDM or any signs of glucose intolerance or newborn's macrosomia in any earlier pregnancy; type 1 or 2 diabetes in first- or second-degree relatives; or age $>40$ years old

Participants Exclusion criteria: $\geq 1$ of the three baseline (8-12 week gestation) oral glucose tolerance test abnormal measurements (fasting blood glucose $>5.3 \mathrm{mmol} / \mathrm{l}, .10 .0 \mathrm{mmol} / \mathrm{l}$ at $1 \mathrm{~h}$, and $.8 .6 \mathrm{mmol} / \mathrm{l}$ at $2 \mathrm{~h}$ ); prepregnant type 1 or 2 diabetes; inability to speak Finnish; $<18$ years old; multiple pregnancy; physical restriction preventing physical activity; substance abuse; treatment or clinical history for psychiatric illness

\begin{tabular}{ll}
\hline & $\begin{array}{l}\text { Intervention: }(\mathrm{n}=219) \text { individual intensified counseling on physical } \\
\text { activity to increase amount of physical activity and diet and weight gain } \\
\text { at five antenatal visits. Goal diet of }<10 \% \text { saturated fat, } 5 \%-10 \% \\
\text { polyunsaturated fat, } 25 \%-30 \% \text { total fat, and } 10 \% \text { saccharide of total } \\
\text { energy intake, and } 25-35 \mathrm{~g} / \mathrm{d} \text { fiber. Participants made individual goals. } \\
\text { Control: }(\mathrm{n}=180)\end{array}$ \\
\hline & $\begin{array}{l}\text { Intervention group GWG: } 13.8 \pm 5.8 \mathrm{~kg} \\
\text { Control group GWG: } 14.2 \pm 5.1 \mathrm{~kg} \\
\text { p-value: } 0.52\end{array}$ \\
\hline
\end{tabular}

McGowan et al. 2013

\begin{tabular}{|c|c|}
\hline Methods & $\begin{array}{l}\text { RCT } \\
\text { Location: Dublin, Ireland }\end{array}$ \\
\hline Participants & $\begin{array}{l}800 \text { randomized participants } \\
\text { Inclusion criteria: } \geq 18 \text { years, singleton; } 10-18 \text { weeks gestation; adequate } \\
\text { English to enable study participation } \\
\text { Exclusion criteria: previous or current gestational diabetes (GDM); } \\
\text { taking medication for a known medical condition; multiple pregnancy }\end{array}$ \\
\hline Intervention & $\begin{array}{l}\text { Intervention: ( } n=235) \text { 1-2 hours GI-dietary education session in groups } \\
2-6 \text { with dietitian for at least } 2 \text { weeks based on Irish Nutrition and } \\
\text { Dietetic Institute; not given specific information on their individual } \\
\text { energy requirements or GWG goal for pregnancy; given list of foods that } \\
\text { were high and low in GI; compliance questionnaire; 3-day food diary } \\
\text { Control: }(n=285)\end{array}$ \\
\hline Results & $\begin{array}{l}\text { Intervention group GWG: } 11.5 \pm 4.2 \mathrm{~kg} \\
\text { Control group GWG: } 12.6 \pm 4.4 \mathrm{~kg} \\
\text { p-value: } 0.003\end{array}$ \\
\hline
\end{tabular}

Fig. 2 (Continued) 
Mustila et al. 2012

\begin{tabular}{|c|c|}
\hline \multirow{2}{*}{ Methods } & RCT \\
\hline & Location: Tampere and Hämeenlinna, Finland \\
\hline Participants & $\begin{array}{l}72 \text { randomized participants } \\
\text { Inclusion criteria: no previous deliveries } \\
\text { Exclusion criteria: <18 years, type } 1 \text { or type } 2 \text { diabetes mellitus } \\
\text { (gestational diabetes mellitus excluded); twin pregnancy; physical } \\
\text { disability preventing exercising; otherwise problematic pregnancy } \\
\text { (determined by a physician); substance abuse, treatment or clinical } \\
\text { history for any psychiatric illness; inadequate language skills in Finnish; } \\
\text { intention to change residence within three months }\end{array}$ \\
\hline Intervention & $\begin{array}{l}\text { Intervention: ( } n=35) \text { individual counseling on physical activity and diet } \\
\text { at five routine visits to a maternity health care nurse starting at } 8-9 \\
\text { weeks of gestation. Option to attend supervised group exercise sessions } \\
\text { once a week during pregnancy until } 37 \text { weeks' gestation. } \\
\text { Control: }(n=38)\end{array}$ \\
\hline Results & $\begin{array}{l}\text { Intervention group GWG: } 14.1 \pm 4.5 \mathrm{~kg} \\
\text { Control group GWG: } 13.6 \pm 5.1 \mathrm{~kg} \\
\text { p-value: } 0.69\end{array}$ \\
\hline
\end{tabular}

Nascimento et al. 2011

\begin{tabular}{ll}
\hline Methods & $\begin{array}{l}\text { RCT, clinical } \\
\text { Location: Prenatal Outpatient Clinic of the Women's Integral } \\
\text { Healthcare Centre, University of Campinas, Campinas, Brazil }\end{array}$ \\
\hline & 82 randomized participants \\
Inclusion criteria: BMI $26.0-29.9 \mathrm{~kg} / \mathrm{m} 2$ or $\geq 30.0 \mathrm{~kg} / \mathrm{m} 2 ; \geq 18$ years, $14-$ \\
\\
24 weeks gestation \\
Exclusion criteria: multiple gestations; exercising regularly and \\
conditions that contraindicate exercise; risk of abortion
\end{tabular}

Fig. 2 (Continued) 
e106 Nutrition and Exercise Strategies to Prevent Excessive GWG Craemer et al.

\begin{tabular}{ll}
\hline & Intervention groups GWG: \\
& Overall: $10.3 \pm 5.0 \mathrm{~kg}$ \\
& OW: $10.0 \pm 1.7 \mathrm{~kg}$ \\
& OB: $10.4 \pm 5.6 \mathrm{~kg}$ \\
& Control groups GWG: \\
& Overall: $11.5 \pm 7.4 \mathrm{~kg}$ \\
OV: $16.4 \pm 3.9 \mathrm{~kg}$ \\
OB: $10.9 \pm 7.6 \mathrm{~kg}$ \\
p-value Overall: 0.543 \\
p-value OV: 0.001 \\
p-value OB: 0.757 \\
\hline
\end{tabular}

Petrella et al. 2014

\section{RCT}

Methods Location: Obstetric Unit, Mother-Infant Dept. of Policlinico Hospital University of Modena, Italy

63 randomized participants

Inclusion criteria: pre-pregnancy BMI $\geq 25 \mathrm{~kg} / \mathrm{m} 2$, age $>18$ years, singleton

Participants

Exclusion criteria: twin pregnancy; chronic diseases; gestational diabetes mellitus in previous pregnancies; smoking; previous bariatric surgery; regular physical activity; dietary supplements or herbal products known to affect body weight; medical conditions that might affect body weight; plans to deliver outside our Birth Center

Intervention: $(n=33)$ therapeutic life changes participants given diet based on $\mathrm{BMI}$ and recommended mild physical activity of 30 minutes Intervention per day, 3 times per week. Overweight participants with $1700 \mathrm{kcal} / \mathrm{day}$, obese participants with $1800 \mathrm{kcal} /$ day.

Control: $(n=30)$

Intervention group GWG: $8.8 \pm 6.5 \mathrm{~kg}$

Results

Control group GWG: $10.4 \pm 5.0 \mathrm{~kg}$

p-value: 0.032

Phelan et al. 2011

\begin{tabular}{ll}
\hline Methods & RCT \\
& Location: Providence, Rhode Island
\end{tabular}

Fig. 2 (Continued) 
Participants

338 randomized participants

Inclusion criteria: gestational age 10 to 16 weeks, BMI 19.8 to 40 , nonsmoking, adults (aged $>18$ years), fluency in English, access to a telephone, singleton

Exclusion criteria: major health or psychiatric diseases, weight loss during pregnancy, or a history of $>3$ miscarriages

Intervention: $(n=179)$ behavior intervention with counseling to promote changes in eating and physical activity with one face-to-face visit.

Recommended 30 min of walking most days of the week, and calorie

Intervention goals $(20 \mathrm{kcal} / \mathrm{kg}$ ). Self-monitoring with provided body-weight scales, food records, and pedometers. Given personalized graphs of their weight gains with feedback at each appointment. Additional support for women not in IOM range.

Control: $(n=184)$

Intervention groups GWG:

NW: $15.3 \mathrm{~kg}$

OV/OB: $14.7 \mathrm{~kg}$

Results Control groups GWG:

$\mathrm{NW}: 16.2 \mathrm{~kg}$

OW/OB: $15.1 \mathrm{~kg}$

p-value NW: 0.003

p-value OV/OB: 0.33

Poston et al. 2015

Methods

RCT, March 31, 2009-June 2, 2014

Location: Eight hospitals in multi-ethnic, inner-city UK

1555 randomized participants

Inclusion criteria: $15-18$ weeks gestation; $>16$ years, $\mathrm{BMI} \geq 30 \mathrm{~kg} / \mathrm{m} 2$

Exclusion criteria: unwilling or unable to give informed consent; underlying disorders, including a pre-pregnancy diagnosis of essential

Participants hypertension, diabetes, renal disease, systemic lupus erythematosus, antiphospholipid syndrome, sickle cell disease, thalassemia, coeliac disease, thyroid disease, and current psychosis; or if currently being prescribed metformin

Intervention: ( $n=526)$ behavior intervention with 1 hour/once per week for 8 weeks with health trainer. Food frequency questionnaire before trial. Made SMART goals, given nutrition and exercise

Intervention recommendations. Exercise based on International Physical Activity Questionnaire. Given exercise DVD and log book.

Control: $(n=567)$

Fig. 2 (Continued) 
e108 Nutrition and Exercise Strategies to Prevent Excessive GWG Craemer et al.

Intervention group GWG: $7.2 \pm 4.6 \mathrm{~kg}$

Results

Control group GWG: $7.8 \pm 4.6 \mathrm{~kg}$

p-value: 0.041

Quinlivan et al. 2011

\begin{tabular}{|c|c|}
\hline Methods & $\begin{array}{l}\text { RCT } \\
\text { Location: Melbourne, Australia }\end{array}$ \\
\hline Participants & $\begin{array}{l}126 \text { randomized participants } \\
\text { Inclusion criteria: pregnant with a fetus with no known anomalies, spoke } \\
\text { English, did not intend to relinquish their infant, singleton, able to } \\
\text { attend hospital for antenatal care and were overweight (BMI 25-29.9) } \\
\text { or obese (BMI >29.9) } \\
\text { Exclusion criteria: NR }\end{array}$ \\
\hline Intervention & $\begin{array}{l}\text { Intervention: ( } n=63 \text { ) participants weighed each visit, } 5 \text {-minute } \\
\text { intervention by food technologist, info on reading food labels, shopping } \\
\text { lists of affordable foods available from local shops and healthy recipes, } \\
\text { clinical psychology management. } \\
\text { Control: }(n=61)\end{array}$ \\
\hline Results & $\begin{array}{l}\text { Intervention group GWG: } 7.0 \text { kg } \\
\text { Control group GWG: } 13.8 \mathrm{~kg} \\
\text { p-value: }<0.001\end{array}$ \\
\hline
\end{tabular}

Rauh et al. 2013

\begin{tabular}{ll}
\hline Methods & Cluster RCT \\
& Location: Munich, Germany \\
\hline & 224 randomized participants \\
& Inclusion criteria: $>18$ years; singleton; $<18$ weeks; BMI: $\geq 18.5 \mathrm{~kg} / \mathrm{m} 2$, \\
& and "sufficient" German \\
& Exclusion criteria: any condition preventing physical activity; pre- \\
& pregnancy diabetes; uncontrolled chronic diseases that may affect \\
& weight development \\
Participants & Intervention: ( $\mathrm{n}=152)$ two individual counseling sessions on diet, \\
& physical activity, and weight monitoring with food log. Advised to \\
& exercise 30 minutes of moderate intensity activity most days of the \\
& week at appropriate heart-rate zone and do non-weight-bearing or low- \\
& impact endurance exercises using large muscle groups. Provided with \\
& local prenatal exercise programs list. \\
& Control: $(n=72)$
\end{tabular}

Fig. 2 (Continued) 
Intervention group GWG: $14.1 \pm 4.1 \mathrm{~kg}$

Results Control group GWG: $15.6 \pm 5.8 \mathrm{~kg}$

p-value: 0.035

Renault et al. 2014

\begin{tabular}{|c|c|}
\hline Methods & $\begin{array}{l}\text { RCT } \\
\text { Location: Hvidovre Hospital, University of Copenhagen }\end{array}$ \\
\hline Participants & $\begin{array}{l}389 \text { randomized participants } \\
\text { Inclusion criteria: BMI }>30 ;>18 \text { years; singleton and normal scan at } \\
\text { weeks } 11-14 ;<16 \text { weeks gestation; ability to read and speak Danish } \\
\text { Exclusion criteria: multiple pregnancy; pre-gestational diabetes; other } \\
\text { serious diseases limiting their level of physical activity; previous bariatric } \\
\text { surgery, or alcohol or drug abuse }\end{array}$ \\
\hline Intervention & $\begin{array}{l}\text { Intervention 1: }(n=142) \text { unsupervised exercise with goal of } 11,000 \text { steps } \\
\text { per day using pedometer registered on } 7 \text { consecutive days every } 4 \\
\text { weeks. Reminding text message when a recording period started. Met } \\
\text { with dietitian every } 2 \text { weeks, alternating between outpatient visits and } \\
\text { phone contacts. } \\
\text { Intervention 2: }(n=142) \text { unsupervised exercise with goal of } 11,000 \text { steps } \\
\text { per day using pedometer registered on } 7 \text { consecutive days every } 4 \\
\text { weeks. Reminding text message when a recording period started. } \\
\text { Control: }(n=141)\end{array}$ \\
\hline Results & $\begin{array}{l}\text { Intervention group } 1 \mathrm{GWG}: 8.6 \mathrm{~kg} \\
\text { Intervention group } 2 \mathrm{GWG}: 9.4 \mathrm{~kg} \\
\text { Control group GWG: } 10.9 \mathrm{~kg} \\
\text { p-value I1 vs C: } 0.008 \\
\text { p-value I2 vs C: } 0.042 \\
\text { p-value I1 vs I2: } 0.57\end{array}$ \\
\hline
\end{tabular}

Ruchat et al. 2012

\begin{tabular}{ll}
\hline Methods & RCT \\
& Location: London, Ontario, Canada \\
\hline & 73 randomized participants \\
Inclusion criteria: not have participated in any structured exercise \\
program during pregnancy; BMI 18.5-24.9; $16-20$ weeks gestation \\
Exclusion criteria: $<18$ years or $>40$ years; smoking; multiple pregnancy; \\
presence of chronic disease, or other contraindications to exercise
\end{tabular}

Fig. 2 (Continued) 
e110 Nutrition and Exercise Strategies to Prevent Excessive GWG Craemer et al.

Intervention 1: $(n=23)$ supervised low intensity exercise walking sessions 3-4 times per week, gradually increasing time 25 to 40 minutes. Wore HR monitor. Modified gestational diabetes meal plan.

Intervention Intervention 2: $(n=26)$ supervised moderate intensity exercise walking sessions 3-4 times per week, gradually increasing time 25 to 40 minutes. Wore HR monitor. Modified gestational diabetes meal plan.

Control: $(n=45)$

Intervention group $1 \mathrm{GWG}: 15.3 \pm 2.9 \mathrm{~kg}$

Intervention group $2 \mathrm{GWG}: 14.9 \pm 3.8 \mathrm{~kg}$

Results Control group GWG: $18.3 \pm 5.3 \mathrm{~kg}$

p-value C vs I1: 0.01

p-value C vs 12: 0.003

p-value I1 vs I2: 0.72

Ruiz et al. 2013

\begin{tabular}{ll}
\hline Rethods & RCT, Sept 1, 2007-Jan 31, 2011 \\
& Location: Madrid, Spain \\
\hline & 962 randomized participants \\
Inclusion criteria: sedentary (exercising for $<20$ minutes on $<3$ \\
days/week); singleton; uncomplicated gestation; not at high risk of \\
preterm delivery; not participating in any other trial \\
Exclusion: any obstetric contraindication to exercise \\
\\
Intervention: (n= 481) supervised exercise program with light to \\
moderate-intensity aerobic and resistance exercises 3 days $/$ week (50-55 \\
min/session). Heart rate was consistently less than $60 \%$ of their age- \\
predicted maximum heart rate. Exercises included resistance, core and \\
cardio. \\
Control: $(n=481)$ \\
Intervention groups GWG: \\
NW: $12.3 \pm 3.6 \mathrm{~kg}$ \\
OV/OB: $11.1 \pm 4.3 \mathrm{~kg}$ \\
Control groups GWG: \\
NW: $13.8 \pm 4.1 \mathrm{~kg}$ \\
OV/OB: $11.6 \pm 4.2 \mathrm{~kg}$ \\
$\mathrm{p}$-value Overall: $<0.001$ \\
$\mathrm{p}$-value NW: $<0.001$ \\
$\mathrm{p}$-value OV/OB: 0.51 \\
\\
\hline
\end{tabular}

\section{Szmeja et al. 2014}

Fig. 2 (Continued) 
Methods

-

Randomized parallel trial

Location: Australia and New Zealand

1108 randomized participants

Participants

Inclusion criteria: singleton pregnancy; 10 -20 weeks gestation; $\mathrm{BMI} \geq 25$

$\mathrm{kg} / \mathrm{m} 2$

Exclusion criteria: NR

Intervention: ( $n=534$ ) informational DVD on healthy eating, serving sizes, and exercise during pregnancy. Individualized advice for balance of carbohydrates, fat and protein, reduce intake of foods high in refined

Intervention carbohydrates, saturated fats and increase intake of fiber and suggested two servings of fruit, five servings of vegetables, and three servings of dairy each day. Increase walking and incidental activity. Session with RD, set goals and self-monitor progress.

Control: $(n=565)$

Intervention group GWG: $9.1 \pm 5.8 \mathrm{~kg}$

Results Control group GWG: $9.7 \pm 5.7 \mathrm{~kg}$

p-value: 0.13

Tanvig et al. 2015

\begin{tabular}{ll}
\hline Methods & RCT \\
& Location: Odense and Aarhus University Hospitals, Denmark \\
\hline & 150 randomized participants \\
Inclusion criteria: singleton; born at term in 2008 to 2009; BMI 18.5- & $24.9 \mathrm{~kg} / \mathrm{m} 2$; healthy Caucasian \\
Exclusion criteria: NR
\end{tabular}

Vesco et al. 2015

Methods

parallel group RCT

Location: Kaiser Permanente, Northwest Oregon

Fig. 2 (Continued) 
e112 Nutrition and Exercise Strategies to Prevent Excessive GWG Craemer et al.

114 randomized participants

Inclusion criteria: English-speaking; $\mathrm{BMI} \geq 30 \mathrm{~kg} / \mathrm{m} 2$; $\geq 18$ years; receiving prenatal care at Kaiser Permanente, Northwest

Participants Exclusion criteria: diabetes mellitus or other medical conditions requiring specialized nutritional care; plans to leave area during follow up period

Intervention: ( $n=56$ ) participants given dietary approaches to stop hypertension dietary pattern without sodium restriction, and weekly

Intervention group meetings. Instructed to exercise 30 minutes per day.

Control: $(n=58)$

Intervention group GWG: $5.0 \pm 4.1 \mathrm{~kg}$

Results Control group GWG: $8.4 \pm 4.7 \mathrm{~kg}$ p-value: $<0.001$

Walsh et al. 2014

\begin{tabular}{ll}
\hline Methods & RCT \\
& Location: Dublin, Ireland \\
\hline & $\begin{array}{l}\text { } 00 \text { randomized participants } \\
\text { Inclusion criteria: } 2 \text { nd pregnancy } \\
\text { Exclusion criteria: any underlying medical disorders; history of } \\
\text { gestational diabetes; using medications; unable to give full informed } \\
\text { consent }\end{array}$ \\
\hline Intervention: (n= 235) 2-hour dietary education session in groups of 2-6 \\
women with dietician. 3-day food diary. First advised on general healthy \\
eating guidelines, then focused on Gl. Received resources on LGI foods. \\
Advice not given for LGI recommendations for GWG. \\
Control: (n=285) \\
Intervention & Control group GWG: $13.7 \pm 4.9 \mathrm{~kg}$ \\
& p-value: 0.01 \\
\hline
\end{tabular}

Fig. 2 (Continued) 
Table 1 Comparison of GWG $(\mathrm{kg})$

\begin{tabular}{|c|c|c|c|c|}
\hline \multirow[t]{2}{*}{ Study or subgroup } & \multicolumn{2}{|c|}{ Intervention } & \multicolumn{2}{|c|}{ Control } \\
\hline & Mean & SD & Mean & SD \\
\hline \multicolumn{5}{|l|}{ Nutrition } \\
\hline Di Carlo et al (2014) & 8.2 & 4.0 & 13.4 & 4.2 \\
\hline Horan et al (2016) & 13.3 & 4.5 & 13.7 & 4.9 \\
\hline Luo et al (2014) & 7.6 & 1.6 & 12.6 & 4.6 \\
\hline McGowan et al (2013) & 11.5 & 4.2 & 12.6 & 4.4 \\
\hline Quinlivan et al (2011) & 7.0 & NR & 13.8 & NR \\
\hline \multirow[t]{2}{*}{ Walsh and McAuliffe (2015) } & 12.2 & 4.4 & 13.7 & 4.9 \\
\hline & \multicolumn{2}{|c|}{ p-Value: 0.013} & & \\
\hline \multicolumn{5}{|l|}{ Nutrition plus exercise } \\
\hline Althuizen et al (2013) & 11.1 & 3.2 & 11.6 & 4.6 \\
\hline Asbee et al (2009) & 13.0 & 5.7 & 16.2 & 7.0 \\
\hline Aşcı and Rathfisch (2016) & 12.5 & 5.0 & 12.3 & 4.8 \\
\hline Bogaerts et al (2013) (1) & 9.5 & 6.8 & 13.5 & 7.3 \\
\hline Bogaerts et al (2013) (2) & 10.6 & 7.0 & 13.5 & 7.3 \\
\hline Huang et al (2011) & 14.0 & 2.4 & 16.2 & 3.3 \\
\hline Hui et al (2012) & 14.1 & 6.0 & 15.2 & 5.9 \\
\hline Hui et al (2014) & 12.9 & 3.7 & 16.2 & 4.4 \\
\hline Korpi-Hyövälti et al (2011) & 11.4 & 6.0 & 13.9 & 5.1 \\
\hline Luoto et al (2011) & 13.8 & 5.8 & 14.2 & 5.1 \\
\hline Mustila et al (2012) & 13.6 & 5.1 & 14.1 & 4.5 \\
\hline Petrella et al (2014) & 8.8 & 6.5 & 10.4 & 5.0 \\
\hline Phelan et al (2011) (NW) & 15.3 & 4.4 & 16.2 & 4.6 \\
\hline Phelan et al (2011) (OV/OB) & 14.7 & 6.9 & 15.1 & 7.5 \\
\hline Poston et al (2015) & 7.2 & 4.6 & 7.8 & 4.6 \\
\hline Rauh et al (2013) & 14.1 & 4.1 & 15.6 & 5.8 \\
\hline Renault et al (2014) & 8.6 & NR & 10.9 & NR \\
\hline Ruchat et al (2012) (LI) & 15.3 & 2.9 & 18.3 & 5.3 \\
\hline Ruchat et al (2012) (MI) & 14.9 & 3.8 & 18.3 & 5.3 \\
\hline Szmeja et al (2014) & 9.1 & 5.8 & 9.7 & 5.7 \\
\hline Tanvig et al (2015) & 7.0 & NR & 8.8 & NR \\
\hline \multirow[t]{2}{*}{ Vesco et al (2014) } & 5.0 & 4.1 & 8.4 & 4.7 \\
\hline & \multicolumn{2}{|c|}{ p-Value: 0.056} & & \\
\hline \multicolumn{5}{|l|}{ Exercise } \\
\hline Byrne et al (2011) & 10.8 & 5.1 & 11.8 & 5.9 \\
\hline Garnæs et al (2016) & 10.5 & NR & 9.2 & NR \\
\hline Haakstad and Bø (2011) & 13.0 & 4.0 & 13.8 & 4.0 \\
\hline Kong et al (2014) (OV) & 10.5 & 5.4 & 9.9 & 6.1 \\
\hline Kong et al (2014) (OB) & 12.1 & 9.0 & 12.5 & 8.5 \\
\hline Nascimento et al (2011) & 10.3 & 1.7 & 16.4 & 3.9 \\
\hline Nascimento et al (2011) (OV) & 10.0 & 1.7 & 16.4 & 3.9 \\
\hline Nascimento et al (2011) (OB) & 10.4 & 5.6 & 10.9 & 7.6 \\
\hline Renault et al (2014) & 9.4 & NR & 10.9 & NR \\
\hline Ruiz et al (2013) (NW) & 12.3 & 3.6 & 13.8 & 4.1 \\
\hline \multirow[t]{2}{*}{ Ruiz et al (2013) (OV/OB) } & 11.1 & 4.3 & 11.6 & 4.2 \\
\hline & \multicolumn{2}{|c|}{ p-Value: 0.069} & & \\
\hline
\end{tabular}

(Continued)
Table 1 (Continued)

\begin{tabular}{|c|c|c|c|c|}
\hline \multirow[t]{2}{*}{ Study or subgroup } & \multicolumn{2}{|c|}{ Intervention } & \multicolumn{2}{|c|}{ Control } \\
\hline & Mean & SD & Mean & SD \\
\hline \multicolumn{5}{|l|}{ Supervised exercise } \\
\hline Garnæs et al (2016) & 10.5 & 10.5 & 9.2 & NR \\
\hline Haakstad and Bø (2011) & 13.0 & 4.0 & 13.8 & 4.0 \\
\hline Nascimento et al (2011) & 10.3 & 1.7 & 16.4 & 3.9 \\
\hline Nascimento et al (2011) (OV) & 10.0 & 1.7 & 16.4 & 3.9 \\
\hline Nascimento et al (2011) (OB) & 10.4 & 5.6 & 10.9 & 7.6 \\
\hline Ruiz et al (2013) (NW) & 12.3 & 3.6 & 13.8 & 4.1 \\
\hline \multirow[t]{2}{*}{ Ruiz et al (2013) (OV/OB) } & 11.1 & 4.3 & 11.6 & 4.2 \\
\hline & \multicolumn{2}{|c|}{ p-Value: 0.61} & & \\
\hline \multicolumn{5}{|l|}{ Unsupervised exercise } \\
\hline Byrne et al (2011) & 10.8 & 5.1 & 11.8 & 5.9 \\
\hline Kong et al (2014) (OV) & 10.5 & 5.4 & 9.9 & 6.1 \\
\hline Kong et al (2014) (OB) & 12.1 & 9.0 & 12.5 & 8.5 \\
\hline \multirow[t]{3}{*}{ Renault et al (2014) } & 9.4 & NR & 10.9 & NR \\
\hline & \multicolumn{2}{|c|}{ p-Value: 0.494} & & \\
\hline & \multicolumn{2}{|c|}{$\begin{array}{l}\text { Total p-value: } \\
0.0014\end{array}$} & & \\
\hline
\end{tabular}

Abbreviations: GWG, gestational weight gain; LI, low intensity; MI, moderate intensity; NR not reported; NW, normal weight; OB, obesity; OV, overweight; SD, standard deviation.

Table 2 Comparison of GWG $(\mathrm{kg})$ for studies that selected participants who are OV or have OB based on mean pre-pregnancy BMI

\begin{tabular}{|l|l|l|l|l|}
\hline \multirow{2}{*}{ Study or subgroup } & \multicolumn{3}{l|}{ Intervention } & \multicolumn{2}{l|}{ Control } \\
\cline { 2 - 5 } & Mean & SD & Mean & SD \\
\hline Nutrition & 8.2 & 4.0 & 13.4 & 4.2 \\
\hline Di Carlo et al (2014) & 7.0 & NR & 13.8 & NR \\
\hline Quinlivan et al (2011) & \multicolumn{5}{|l|}{-Value: 0.011} & & \\
\hline \multicolumn{5}{|l|}{} \\
\hline Nutrition plus exercise & 9.5 & 6.8 & 13.5 & 7.3 \\
\hline Bogaerts et al (2013) (1) & 10.6 & 7.0 & 13.5 & 7.3 \\
\hline Bogaerts et al (2013) (2) & 11.4 & 6.0 & 13.9 & 5.1 \\
\hline Korpi-Hyövälti et al (2011) & 11.9 & 5.8 & 14.2 & 5.1 \\
\hline Luoto et al (2011) & 13.8 & 8.2 & 5.0 \\
\hline Petrella et al (2014) & 8.8 & 6.5 & 10.4 & 7.5 \\
\hline Phelan et al (2011) (OV/OB) & 14.7 & 6.9 & 15.1 & 7.6 \\
\hline Poston et al (2015) & 7.2 & 4.6 & 7.8 & 4.6 \\
\hline Renault et al (2014) & 8.6 & NR & 10.9 & NR \\
\hline Szmeja et al (2014) & 9.1 & 5.8 & 9.7 & 5.7 \\
\hline Tanvig et al (2015) & 7.0 & NR & 8.8 & NR \\
\hline Vesco et al (2014) & 5.0 & 4.1 & 8.4 & 4.7 \\
\hline & $p$-Value: 0.129 & & \\
\hline
\end{tabular}

(Continued) 
Table 2 (Continued)

\begin{tabular}{|c|c|c|c|c|}
\hline \multirow[t]{2}{*}{ Study or subgroup } & \multicolumn{2}{|c|}{ Intervention } & \multicolumn{2}{|c|}{ Control } \\
\hline & Mean & SD & Mean & SD \\
\hline \multicolumn{5}{|l|}{ Exercise } \\
\hline Byrne et al (2011) & 10.8 & 5.1 & 11.8 & 5.9 \\
\hline Garnæs et al (2016) & 10.5 & NR & 9.2 & NR \\
\hline Kong et al (2014) (OV) & 10.5 & 5.4 & 9.9 & 6.1 \\
\hline Kong et al (2014) (OB) & 12.1 & 9.0 & 12.5 & 8.5 \\
\hline \multirow[t]{2}{*}{ Nascimento et al (2011) } & 10.3 & 1.7 & 16.4 & 3.9 \\
\hline & \multicolumn{4}{|c|}{$\begin{array}{l}\text { Does not differentiate between } \\
\text { OV and obese }\end{array}$} \\
\hline \multirow[t]{2}{*}{ Renault et al (2014) } & 9.4 & NR & 10.9 & NR \\
\hline & \multicolumn{2}{|c|}{$\begin{array}{l}p \text {-Value: } \\
0.308\end{array}$} & & \\
\hline \multicolumn{5}{|l|}{ Supervised exercise } \\
\hline Garnæs et al (2016) & 10.5 & NR & 9.2 & NR \\
\hline \multirow[t]{2}{*}{ Nascimento et al (2011) } & 10.3 & 1.7 & 16.4 & 3.9 \\
\hline & \multicolumn{2}{|c|}{$\begin{array}{l}p \text {-Value: } \\
0.575\end{array}$} & & \\
\hline \multicolumn{5}{|l|}{ Unsupervised exercise } \\
\hline Byrne et al (2011) & 10.8 & 5.1 & 11.8 & 5.9 \\
\hline Kong et al (2014) (OV) & 10.5 & 5.4 & 9.9 & 6.1 \\
\hline Kong et al (2014) (OB) & 12.1 & 9.0 & 12.5 & 8.5 \\
\hline \multirow[t]{3}{*}{ Renault et al (2014) } & 9.4 & NR & 10.9 & NR \\
\hline & \multicolumn{2}{|c|}{$\begin{array}{l}p \text {-Value: } \\
0.494\end{array}$} & & \\
\hline & \multicolumn{2}{|c|}{$\begin{array}{l}\text { Total p-value: } \\
0.0055\end{array}$} & & \\
\hline
\end{tabular}

Abbreviations: BMI, body mass index; GWG, gestational weight gain; LI, low intensity; MI, moderate intensity; NR not reported; NW, normal weight; OB, obesity; OV, overweight; SD, standard deviation.

nutrition plus exercise (70\%), and 1 of 6 exercise only produced significant results (16.67\%). One of two supervised and one of four unsupervised exercise produced significant results. Like the overall analyses, nutrition-only strategy was significant $(p=0.011)$. Overall, comparison between all interventions and controls was significant $(p=0.004)$ in this population.

\section{Exercise Interventions}

Three studies reported statistically significant differences in mean $\mathrm{GWG}^{51,60,61}$ ( - Table 3). Five of eight studies subjects selected for pre-pregnancy BMI as obese or OV. ${ }^{51,56,57,59,60}$ These studies produced GWG greater than IOM recommendation for both control and intervention groups, except when Kong et al (2014) was divided into participants with OV and OB; OV individuals had mean GWG within IOM recommendations. Three studies did not have BMI specifications and were classified as NW with GWG within IOM. ${ }^{52,58,61}$

Studies were then divided into subgroups where exercise was either supervised or unsupervised. To be a supervised intervention, exercise must be performed under the supervision of a personal trainer or by attendance at an exercise class. Neither supervised $(p=0.61)$ nor unsupervised
( $p=0.494)$ programs produced significant results. However, mean GWG for unsupervised intervention $(10.7 \mathrm{~kg})$ and control (11.275 kg) groups was lower than supervised (11.44 kg) intervention and control (12.96 kg) groups.

\section{Nutrition Interventions}

Five of six studies reported statistically significant differences in mean $\mathrm{GWG}^{31,33-36,52}$ (- Table 3). Two studies selected subjects with OV or obese pre-pregnancy BMI. ${ }^{31,35}$ The results of three studies demonstrated that the intervention group's average GWG was within IOM and the average GWG of control group exceed IOM..$^{31,34,35}$ All control groups' GWG exceeded IOM, except one that was within IOM recommendations. ${ }^{34}$ One intervention group's GWG was below IOM recommendations. ${ }^{33}$

\section{Combination of Exercise-Plus-Nutrition Interventions}

Thirteen of 19 studies reported statistically significant differences in mean $\mathrm{GWG}^{38-41,43,47,49-52,54,55}$ (- Table 3). Three underwent multiple analyses. When divided into subgroups, interventions 1 and $2,{ }^{40} \mathrm{NW}$ participants ${ }^{48}$ and both $\mathrm{LI}$ and MI exercise programs were significant. ${ }^{52}$ Ten studies selected participants with OV or obese pre-pregnancy BMI. $^{40,44,45,47-49,51,53-55}$ Of these studies, six interventions and three control groups were within IOM recommendations. All NW subjects produced GWG within the IOM recommendations for the intervention group. ${ }^{37,39,41-43,46,48,50}$

\section{Discussion}

Based on results of this meta-analysis, nutrition-only interventions were associated with statistically significant lower rates of excessive GWG and therefore have the highest probability of helping women achieve target IOM GWG guidelines $(p=0.013)$. Exercise-only $(p=0.069)$ and nutrition-plus-exercise $(p=0.056)$ interventions have potential to control GWG, but results did not reach statistical significance in the current study. In comparison to the Cochrane Review findings of studies published before the 2009 IOM guidelines which found all three intervention groups reduced GWG, the current meta-analysis study found that studies which utilized the 2009 IOM guidelines were more likely to produced reduced GWG with nutrition-only interventions. Nonsignificant findings from exercise and nutrition-plus-exercise health system strategies may be due to the inclusion of different exercise types. Exercise programs varied. Some included advice about exercise or discussed increased amount of physical activity, while others were more interventional, such as supervised dance programs or recommending a certain number of steps per day. The way in which these steps were achieved may and most likely differed between participants. Despite different nutritional advice, interventions universally recommend increased amounts of fruits and vegetables, and decreased consumption of food with high fat and sugar content. Additionally, retention to exercise programs may be more difficult than nutrition programs because one must eat to live, but exercise is not a fundamental need. 


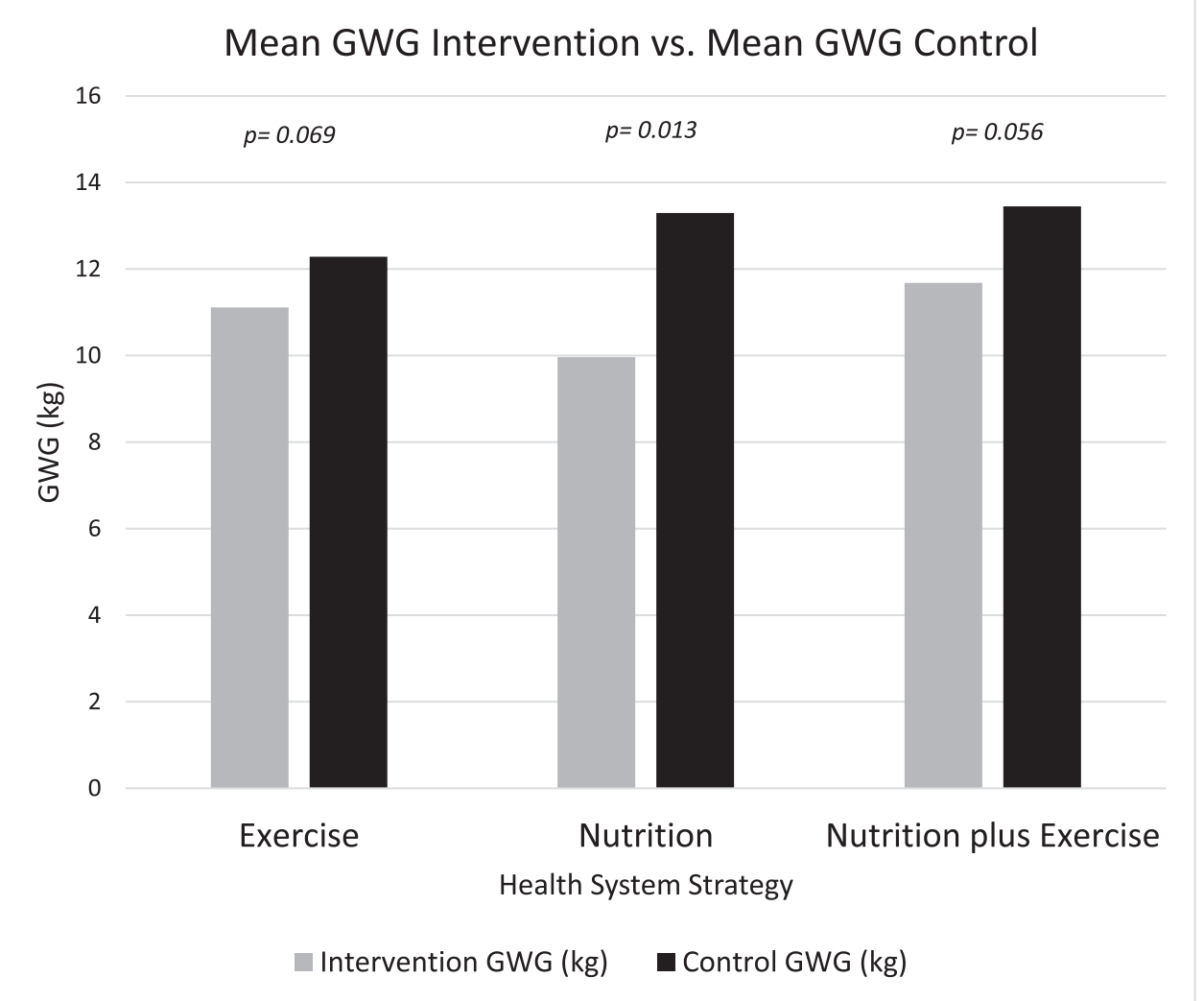

Fig. 3 Mean gestational weight gain (GWG) $(\mathrm{kg})$ of health system strategies intervention groups versus mean GWG control groups.

Table 3 Comparison of mean intervention and control gestational weight gain $(\mathrm{kg})$

\begin{tabular}{|c|c|c|c|c|c|c|c|c|c|}
\hline \multirow[t]{2}{*}{ Study or subgroup } & \multicolumn{3}{|c|}{ Intervention } & \multicolumn{3}{|c|}{ Control } & \multirow[t]{2}{*}{$p$-Value } & \multirow{2}{*}{$\begin{array}{l}\text { Mean } \\
\text { difference }\end{array}$} & \multirow{2}{*}{$\begin{array}{l}\text { IV, random } \\
95 \% \mathrm{Cl}\end{array}$} \\
\hline & $N$ & Mean & SD & $N$ & Mean & SD & & & \\
\hline \multicolumn{10}{|l|}{ Nutrition } \\
\hline Di Carlo et al (2014) & 77 & 8.2 & 4.0 & 77 & 13.4 & 4.2 & $<0.001$ & -5.2 & $-6.51,-3.89$ \\
\hline Horan et al (2016) & 138 & 13.3 & 4.5 & 142 & 13.7 & 4.9 & 0.52 & -0.4 & $-1.51,0.71$ \\
\hline Luo et al (2014) & 131 & 7.6 & 1.6 & 145 & 12.6 & 4.6 & $<0.001$ & -5.0 & $-5.83,-4.17$ \\
\hline McGowan et al (2013) & 235 & 11.5 & 4.2 & 285 & 12.6 & 4.4 & 0.003 & -1.1 & $-1.85,-0.35$ \\
\hline Quinlivan et al (2011) & 63 & 7.0 & NR & 61 & 13.8 & $\mathrm{NR}$ & $<0.001$ & -6.8 & NR \\
\hline \multirow[t]{2}{*}{ Walsh and McAuliffe (2015) } & 235 & 12.2 & 4.4 & 285 & 13.7 & 4.9 & 0.01 & -1.5 & $-2.31,-0.69$ \\
\hline & \multicolumn{9}{|c|}{ Total events: 879 (intervention), 995 (standard care) } \\
\hline \multicolumn{10}{|l|}{ Nutrition and exercise } \\
\hline Althuizen et al (2013) & 106 & 11.1 & 3.2 & 113 & 11.6 & 4.6 & $N R^{a}$ & -0.5 & $-1.56,0.56$ \\
\hline Asbee et al (2009) & 57 & 13.0 & 5.7 & 43 & 16.2 & 7.0 & $<0.01$ & -3.2 & $-5.72,-0.68$ \\
\hline Aşcı and Rathfisch (2016) & 45 & 12.5 & 5.0 & 45 & 12.3 & 4.8 & 0.001 & 0.2 & $-1.85,2.25$ \\
\hline Bogaerts et al (2013) (1) & 58 & 9.5 & 6.8 & 63 & 13.5 & 7.3 & 0.04 & -4.0 & $-6.55,-1.45$ \\
\hline Bogaerts et al (2013) (2) & 76 & 10.6 & 7.0 & 63 & 13.5 & 7.3 & 0.008 & -2.9 & $-5.30,-0.50$ \\
\hline Huang et al (2011) & 61 & 14.0 & 2.4 & 64 & 16.2 & 3.3 & $<0.001$ & -2.2 & $-3.23,-1.17$ \\
\hline Hui et al (2012) & 102 & 14.1 & 6.0 & 88 & 15.2 & 5.9 & 0.28 & -1.1 & $-2.81,0.61$ \\
\hline Hui et al (2014) & 30 & 12.9 & 3.7 & 27.0 & 16.2 & 4.4 & $<0.05$ & -3.3 & $-5.45,-1.15$ \\
\hline Korpi-Hyövälti et al (2011) & 27 & 11.4 & 6.0 & 27.0 & 13.9 & 5.1 & 0.062 & -2.5 & $-5.54,0.54$ \\
\hline Luoto et al (2011) & 219 & 13.8 & 5.8 & 180 & 14.2 & 5.1 & 0.52 & -0.4 & $-1.49,0.96$ \\
\hline Petrella et al (2014) & 33 & 8.8 & 6.5 & 30 & 10.4 & 5.0 & 0.032 & -1.6 & $-4.54,1.34$ \\
\hline Phelan et al (2011) (NW) & 92 & 15.3 & 4.4 & 94 & 16.2 & 4.6 & 0.003 & -0.9 & $-2.20,0.40$ \\
\hline
\end{tabular}


Table 3 (Continued)

\begin{tabular}{|c|c|c|c|c|c|c|c|c|c|}
\hline \multirow[t]{2}{*}{ Study or subgroup } & \multicolumn{3}{|c|}{ Intervention } & \multicolumn{3}{|c|}{ Control } & \multirow[t]{2}{*}{$p$-Value } & \multirow{2}{*}{$\begin{array}{l}\text { Mean } \\
\text { difference }\end{array}$} & \multirow{2}{*}{$\begin{array}{l}\text { IV, random } \\
95 \% \mathrm{Cl}\end{array}$} \\
\hline & $N$ & Mean & SD & $N$ & Mean & SD & & & \\
\hline Phelan et al (2011) (OV/OB) & 87 & 14.7 & 6.9 & 90 & 15.1 & 7.5 & 0.33 & -0.4 & $-2.54,1.74$ \\
\hline Poston et al (2015) & 526 & 7.2 & 4.6 & 567 & 7.8 & 4.6 & 0.041 & -0.6 & $-1.15,-0.05$ \\
\hline Rauh et al (2013) & 152 & 14.1 & 4.1 & 72 & 15.6 & 5.8 & 0.035 & -1.5 & $-2.83,-0.17$ \\
\hline Renault et al (2014) & 142 & 8.6 & NR & 141 & 10.9 & NR & 0.008 & -2.3 & NR \\
\hline Ruchat et al (2012) (LI) & 23 & 15.3 & 2.9 & 45 & 18.3 & 5.3 & 0.01 & -3.0 & $-5.37,-0.63$ \\
\hline Ruchat et al (2012) (MI) & 26 & 14.9 & 3.8 & 45 & 18.3 & 5.3 & 0.003 & -3.4 & $-5.76,-1.04$ \\
\hline Szmeja et al (2014) & 543 & 9.1 & 5.8 & 565 & 9.7 & 5.7 & 0.13 & -0.6 & $-1.28,0.08$ \\
\hline Tanvig et al (2015) & 77 & 7.0 & NR & 73 & 8.8 & NR & 0.01 & -1.8 & NR \\
\hline \multirow[t]{2}{*}{ Vesco et al (2014) } & 56 & 5.0 & 4.1 & 58 & 8.4 & 4.7 & $<0.001$ & -3.4 & $-5.04,-1.76$ \\
\hline & \multicolumn{9}{|c|}{ Total events: 2,538 (intervention), 2,493 (standard care) } \\
\hline \multicolumn{10}{|l|}{ Exercise } \\
\hline Byrne et al (2011) & 12 & 10.8 & 5.1 & 11 & 11.8 & 5.9 & $\mathrm{NR}^{\mathrm{a}}$ & -1.0 & $-5.77,3.77$ \\
\hline Garnæs et al (2016) & 46 & 10.5 & $\mathrm{NR}$ & 45 & 9.2 & NR & 0.35 & -1.3 & NR \\
\hline Haakstad and Bø (2011) & 52 & 13.0 & 4.0 & 53 & 13.8 & 4.0 & 0.31 & -0.8 & $-2.35,0.75$ \\
\hline Kong et al (2014) (OV) & 9 & 10.5 & 5.4 & 9 & 9.9 & 6.1 & 0.859 & 0.6 & $-5.16,6.36$ \\
\hline Kong et al (2014) (OB) & 9 & 12.1 & 9.0 & 10 & 12.5 & 8.5 & 0.859 & -0.4 & $-8.87,8.07$ \\
\hline Nascimento et al (2011) & 39 & 10.3 & 1.7 & 41 & 16.4 & 3.9 & 0.543 & -6.1 & $-7.45,-4.75$ \\
\hline Nascimento et al (2011) (OV) & 9 & 10.0 & 1.7 & 5 & 16.4 & 3.9 & 0.001 & -6.4 & $-9.61,-3.19$ \\
\hline Nascimento et al (2011) (OB) & 30 & 10.4 & 5.6 & 36 & 10.9 & 7.6 & 0.757 & -0.5 & $-3.84,2.84$ \\
\hline Renault et al (2014) & 142 & 9.4 & NR & 141 & 10.9 & NR & 0.042 & -1.5 & NR \\
\hline Ruiz et al (2013) (NW) & 335 & 12.3 & 3.6 & 352 & 13.8 & 4.1 & $<0.001$ & -1.5 & $-2.08,-0.92$ \\
\hline \multirow[t]{2}{*}{ Ruiz et al (2013) (OV/OB) } & 146 & 11.1 & 4.3 & 129 & 11.6 & 4.2 & 0.51 & -0.5 & $-1.51,0.51$ \\
\hline & \multicolumn{9}{|c|}{ Total events: 790 (intervention), 791 (standard care) } \\
\hline \multicolumn{10}{|l|}{ Supervised exercise } \\
\hline Garnæs et al (2016) & 46 & 10.5 & 10.5 & 45 & 9.2 & NR & 0.35 & 1.3 & $\mathrm{NR}$ \\
\hline Haakstad and Bø (2011) & 52 & 13.0 & 4.0 & 53 & 13.8 & 4.0 & 0.31 & -0.8 & $-2.35,0.75$ \\
\hline Nascimento et al (2011) & 39 & 10.3 & 1.7 & 41 & 16.4 & 3.9 & 0.543 & -6.1 & $-7.45,-4.75$ \\
\hline Nascimento et al (2011) (OV) & 9 & 10.0 & 1.7 & 5 & 16.4 & 3.9 & 0.001 & -6.4 & $-9.61,-3.19$ \\
\hline Nascimento et al (2011) (OB) & 30 & 10.4 & 5.6 & 36 & 10.9 & 7.6 & 0.757 & -0.5 & $-3.84,2.84$ \\
\hline Ruiz et al (2013) (NW) & 335 & 12.3 & 3.6 & 352 & 13.8 & 4.1 & $<0.001$ & -1.5 & $-2.08,-0.92$ \\
\hline \multirow[t]{2}{*}{ Ruiz et al (2013) (OV/OB) } & 146 & 11.1 & 4.3 & 129 & 11.6 & 4.2 & 0.51 & -0.5 & $-1.51,0.51$ \\
\hline & \multicolumn{9}{|c|}{ Total events: 657 (intervention), 661 (standard care) } \\
\hline \multicolumn{10}{|l|}{ Unsupervised exercise } \\
\hline Byrne et al (2011) & 12 & 10.8 & 5.1 & 11 & 11.8 & 5.9 & $\mathrm{NR}^{\mathrm{a}}$ & -1.0 & $-5.77,3.77$ \\
\hline Kong et al (2014) (OV) & 9 & 10.5 & 5.4 & 9 & 9.9 & 6.1 & 0.859 & 0.6 & $-5.16,6.36$ \\
\hline Kong et al (2014) (OB) & 9 & 12.1 & 9.0 & 10 & 12.5 & 8.5 & 0.859 & -0.4 & $-8.87,8.07$ \\
\hline \multirow[t]{2}{*}{ Renault et al (2014) } & 142 & 9.4 & NR & 141 & 10.9 & NR & 0.042 & -1.5 & NR \\
\hline & \multicolumn{9}{|c|}{ Total events: 172 (intervention), 171 (standard care) } \\
\hline
\end{tabular}

Abbreviations: $\mathrm{Cl}$, confidence interval; LI, low intensity; MI, moderate intensity; NR not reported; NW, normal weight; OB, obesity; OV, overweight; SD, standard deviation.

${ }^{\mathrm{a} S}$ Stated as insignificant.

Previous studies have addressed the ability to control GWG and prevent weight exceeding IOM recommendations with mixed results. Since the study search for this article was completed, additional studies have been published with inconsistent results. Nutrition-only studies of the general population and of women who are $\mathrm{OV} / \mathrm{OB}$ concur that nutrition-only interventions make significant differences for controlling GWG. ${ }^{6-8}$ Walker et al's (2018) meta-analysis of general population concerning nutrition only, exercise only, and nutrition plus exercise claimed 


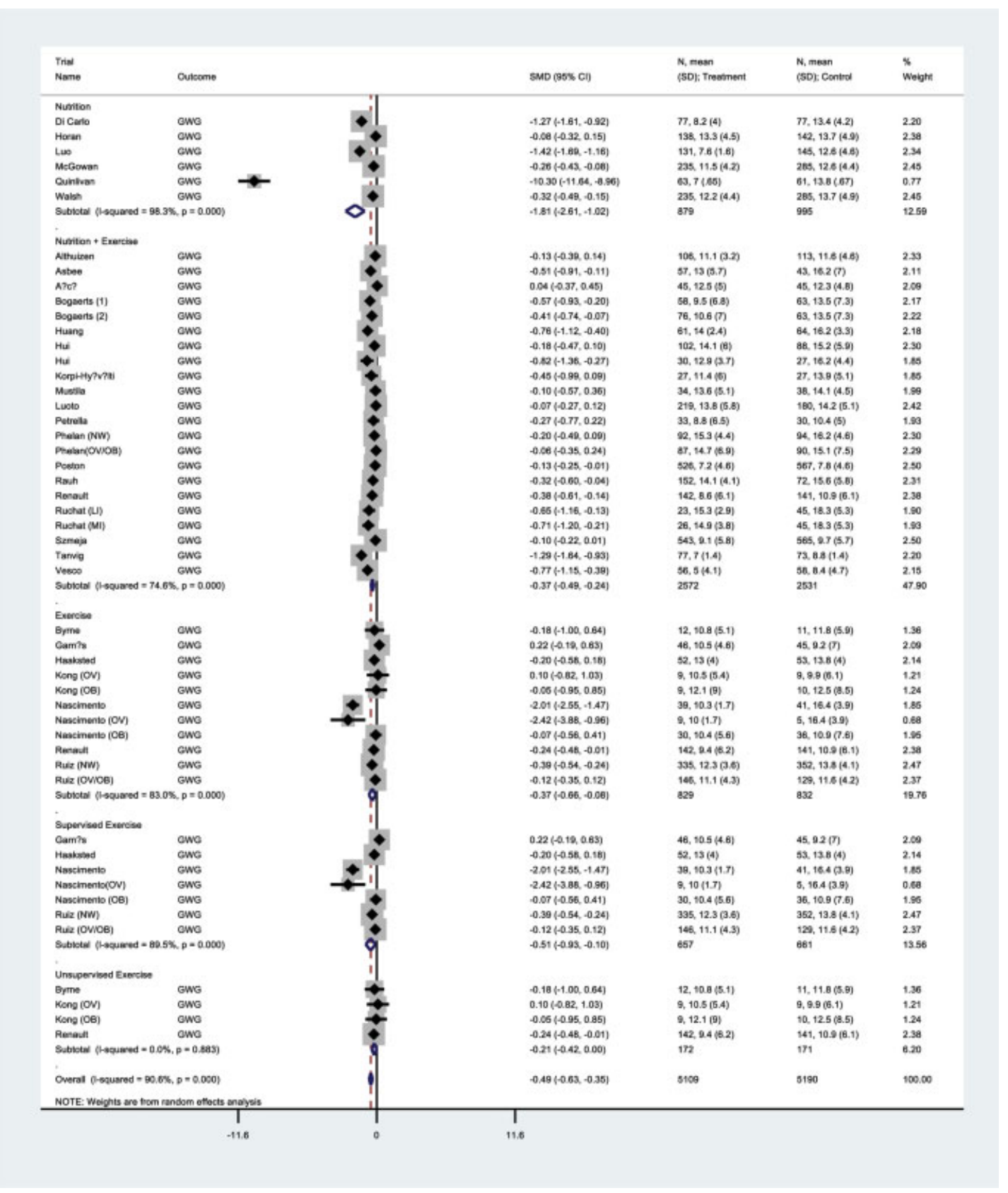

Fig. 4 Forest plot divided by subgroups.

nutrition-only interventions are the best method to control GWG. Lamminpää et al's (2017) meta-analysis of OV/OB women only analyzed nutrition-only interventions because of previous reporting this as the best method. This meta-analysis found that nutrition-only results vary because of adherence inconsistency. Exercise-only interventions produced significant results for both general population $^{10,17,27-29}$ and $\mathrm{OV} / \mathrm{OB}$ except for one study. ${ }^{10}$ Nutrition-plus-exercise interventions for general population were statistically significant. ${ }^{14-21}$ Nutrition plus exercise for $\mathrm{OV} / \mathrm{OB}$ were inconsistent, including a meta-analysis determining nutrition plus exercise as insignificant impact on GWG. ${ }^{20}$ Due to the variability of these findings, it is challenging to make a consensus regarding which inter- vention is best for clinical use. The results of this study confer with other studies that nutrition-only interventions are the best method to control GWG.

\section{Limitations}

Limitations include the inability to calculate risk ratio and weight. Based on heterogeneity of $p$-value greater than 0.10 , 22 studies were determined to have excessive heterogeneity. Heterogeneity between subgroups was not significant; therefore, data were pooled between subgroups. Search did not explore worldwide databases such as EMBASE and LiLACS; therefore, some foreign studies may have been missed that would have otherwise met the inclusion criteria. 
Table 4 Per cent participants that exceed IOM recommendations

\begin{tabular}{|c|c|c|}
\hline Study or subgroup & Intervention & Control \\
\hline \multicolumn{3}{|l|}{ Nutrition } \\
\hline Di Carlo et al (2014) & NR & NR \\
\hline Horan et al (2016) & NR & NR \\
\hline Luo et al (2014) & NR & NR \\
\hline McGowan et al (2013) & 33.2 & 44.7 \\
\hline Quinlivan et al (2011) & NR & NR \\
\hline Walsh and McAuliffe (2015) & 37.7 & 47.9 \\
\hline \multicolumn{3}{|l|}{ Nutrition plus exercise } \\
\hline Althuizen et al (2013) & 70.4 & 72.4 \\
\hline Asbee et al (2009) & 61.4 & 48.8 \\
\hline Aşcı and Rathfisch (2016) & 51.1 & 28.9 \\
\hline Bogaerts et al (2013) (1) & NR & NR \\
\hline Bogaerts et al (2013) (2) & NR & NR \\
\hline Huang et al (2011) & NR & NR \\
\hline Hui et al (2012) & 35.2 & 54.5 \\
\hline Hui et al (2014) & 10 & 37 \\
\hline Korpi-Hyövälti et al (2011) & NR & NR \\
\hline Luoto et al (2011) & NR & NR \\
\hline Mustila et al (2012) & 35.5 & 27.0 \\
\hline Petrella et al (2014) & 33.3 & 60.8 \\
\hline Phelan et al (2011) (NW) & 40.2 & 52.1 \\
\hline Phelan et al (2011) (OV/OB) & 66.7 & 61.1 \\
\hline Poston et al (2015) & NR & NR \\
\hline Rauh et al (2013) & 38.2 & 59.5 \\
\hline Renault et al (2014) & NR & NR \\
\hline Ruchat et al (2012) (LI) & 35 & 53 \\
\hline Ruchat et al (2012) (MI) & 31 & 53 \\
\hline Szmeja et al (2014) & NR & NR \\
\hline Tanvig et al (2015) & NR & NR \\
\hline Vesco et al (2014) & 44 & 82 \\
\hline \multicolumn{3}{|l|}{ Exercise } \\
\hline Byrne et al (2011) & NR & NR \\
\hline Garnæs et al (2016) & 58.3 & 44.4 \\
\hline Haakstad and Bø (2011) & 33 & 38 \\
\hline Kong et al (2014) (OV) & 44.4 & 50 \\
\hline Kong et al (2014) (OB) & 77.8 & 77.8 \\
\hline Nascimento et al (2011) & 47 & 57 \\
\hline Nascimento et al (2011) (OV) & NR & NR \\
\hline Nascimento et al (2011) (OB) & NR & NR \\
\hline Renault et al (2014) & NR & NR \\
\hline Ruiz et al (2013) (NW) & 23.8 & 32 \\
\hline Ruiz et al (2013) (OV/OB) & NR & NR \\
\hline \multicolumn{3}{|l|}{ Supervised exercise } \\
\hline Garnæs et al (2016) & 58.3 & 44.4 \\
\hline Haakstad and Bø (2011) & 33 & 38 \\
\hline Nascimento et al (2011) & 47 & 57 \\
\hline
\end{tabular}

Table 4 (Continued)

\begin{tabular}{|l|l|l|}
\hline Study or subgroup & Intervention & Control \\
\hline Nascimento el al (2011) (OV) & NR & NR \\
\hline Nascimento et al (2011) (OB) & NR & NR \\
\hline Ruiz et al (2013) (NW) & 23.8 & 32 \\
\hline Ruiz et al (2013) (OV/OB) & NR & NR \\
\hline Unsupervised exercise & NR \\
\hline Byrne et al (2011) & 44.4 & NR \\
\hline Kong et al (2014) (OV) & 50 \\
\hline Kong et al (2014) (OB) & 77.8 & 77.8 \\
\hline Renault et al (2014) & NR & NR \\
\hline
\end{tabular}

Abbreviations: IOM, Institute of Medicine; LI, low intensity; MI, moderate intensity; NR not reported; NW, normal weight; OB, obesity; OV, overweight.

Meta-analyses are generally limited due to selection bias and publication bias. No publication bias was detected.

\section{Future Research}

Evidence suggests that nutrition-only, exercise-only, and nutrition-plus-exercise interventions help control excessive GWG during pregnancy. Since the nutrition-only group was the only health system strategy to produce statistically significant results, this type of intervention should be favored as first line. Based on analysis, pre-pregnancy BMI is a better predictor of GWG than the type of intervention program studied. Pregnant individuals with a starting BMI categorized as OV or obese are more likely to exceed IOM recommendations regardless of intervention. Further research is needed on participants that are OV or have $\mathrm{OB}$. Interventions on participants from middleincome or low-income countries should be analyzed because impact of interventions may differ based on several factors.

\section{Conclusion}

We found that nutrition-only interventions were more effective at decreasing rates of excessive GWG than exercise or nutrition-plus-exercise interventions. Time and resources are limited for all clinicians and clinics. Therefore, clinicians and clinical programs should focus efforts on nutrition education with health messages targeting increased consumption of fruits and vegetables and decreased consumption of foods with high fat or sugar content to maximize effectiveness. If additional resources are available, efforts may also be focused on exercise since such efforts are not harmful, but these efforts may be of lower yield.

\section{Funding}

This meta-analysis was not funded.

\section{Conflict of Interest}

The authors of this study declare no conflict of interest. 


\section{References}

1 Ogden CL, Carroll MD, Fryar CD, Flegal KM. Prevalence of obesity among adults and youth: United States, 2011-2014. NCHS Data Brief 2015;219(219):1-8

2 Stang J, Huffman LG. Position of the academy of nutrition and dietetics: obesity, reproduction, and pregnancy outcomes. J Acad Nutr Diet 2016;116(04):677-691

3 Oken E, Taveras EM, Kleinman KP, Rich-Edwards JW, Gillman MW. Gestational weight gain and child adiposity at age 3 years. Am J Obstet Gynecol 2007;196(04):322.e1-322.e8

4 Rasmussen KM, Catalano PM, Yaktine AL. New guidelines for weight gain during pregnancy: what obstetrician/gynecologists should know. Curr Opin Obstet Gynecol 2009;21(06):521-526

5 Gardner B, Wardle J, Poston L, Croker H. Changing diet and physical activity to reduce gestational weight gain: a metaanalysis. Obes Rev 2011;12(07):e602-e620

6 Koutelidakis AE, Alexatou O, Kousaiti S, et al. Higher adherence to Mediterranean diet prior to pregnancy is associated with decreased risk for deviation from the maternal recommended gestational weight gain. Int J Food Sci Nutr 2018;69(01):84-92

7 Walker R, Bennett C, Blumfield M, et al. Attenuating pregnancy weight gain-what works and why: a systematic review and metaanalysis. Nutrients 2018;10(07):944

8 Abdel-Aziz SB, Hegazy IS, Mohamed DA, Abu El Kasem MMA, Hagag SS. Effect of dietary counseling on preventing excessive weight gain during pregnancy. Public Health 2018;154:172-181

9 Quinlivan JA, Julania S, Lam L. Antenatal dietary interventions in obese pregnant women to restrict gestational weight gain to Institute of Medicine recommendations: a meta-analysis. Obstet Gynecol 2011;118(06):1395-1401

10 Shieh C, Cullen DL, Pike C, Pressler SJ. Intervention strategies for preventing excessive gestational weight gain: systematic review and meta-analysis. Obes Rev 2018;19(08):1093-1109

11 Robertson N, Ladlow B. Effect of individual dietetic intervention on gestational weight gain and associated complications in obese pregnant women. Aust N Z J Obstet Gynaecol 2018;58(03):274-277

12 Dorise B, Byth K, McGee T, Wood A, Blumenthal C. A low intensity dietary intervention for reducing excessive gestational weight gain in an overweight and obese pregnant cohort. Eat Weight Disord 2018;•••;. Doi: 10.1007/s40519-018-0566-2

13 Streuling I, Beyerlein A, Rosenfeld E, Hofmann H, Schulz T, von Kries R. Physical activity and gestational weight gain: a metaanalysis of intervention trials. BJOG 2011;118(03):278-284

14 Shepherd E, Gomersall JC, Tieu J, Han S, Crowther CA, Middleton P. Combined diet and exercise interventions for preventing gestational diabetes mellitus. Cochrane Database Syst Rev 2017;11:CD010443

15 Liu YQ Liu Y, Hua Y, Chen XL. Effect of diet and exercise intervention in Chinese pregnant women on gestational weight gain and perinatal outcomes: a quasi-experimental study. Appl Nurs Res 2017;36:50-56

16 Broekhuizen K, Simmons D, Devlieger R, et al. Cost-effectiveness of healthy eating and/or physical activity promotion in pregnant women at increased risk of gestational diabetes mellitus: economic evaluation alongside the DALI study, a European multicenter randomized controlled trial. Int J Behav Nutr Phys Act 2018;15(01):23

17 Nobles C, Marcus BH, Stanek EJ III, et al. The effect of an exercise intervention on gestational weight gain: the Behaviors Affecting Baby and You (B.A.B.Y.) study: a randomized controlled trial. Am J Health Promot 2018;32(03):736-744

18 Chan RS, Tam WH, Ho IC, et al. Randomized trial examining effectiveness of lifestyle intervention in reducing gestational diabetes in high risk Chinese pregnant women in Hong Kong. Sci Rep 2018;8(01):13849

19 Morison PN, Bacardi-Gascon M, Lopez-Corrales M, Jimenez-Cruz A. Combined dietary-exercise intervention for gestational weight gain and birthweight: a meta-analysis. Asia Pac J Clin Nutr 2018; 27(04):860-868
20 Rogozińska E, Marlin N, Betrán AP, et al. Effect of diet and physical activity based interventions in pregnancy on gestational weight gain and pregnancy outcomes: meta-analysis of individual participant data from randomised trials. BMJ 2017; 358:j3119

21 Sagedal LR, Øverby NC, Bere E, et al. Lifestyle intervention to limit gestational weight gain: the Norwegian Fit for Delivery randomised controlled trial. BJOG 2017;124(01):97-109

22 Cahill AG, Haire-Joshu D, Cade WT, et al. Weight control program and gestational weight gain in disadvantaged women with overweight or obesity: a randomized clinical trial. Obesity (Silver Spring) 2018;26(03):485-491

23 Yeo S, Walker JS, Caughey MC, Ferraro AM, Asafu-Adjei JK. What characteristics of nutrition and physical activity interventions are key to effectively reducing weight gain in obese or overweight pregnant women? A systematic review and meta-analysis. Obes Rev 2017;18(04):385-399

24 Van Horn L, Peaceman A, Kwasny M, et al. Dietary approaches to stop hypertension diet and activity to limit gestational weight: maternal offspring metabolics family intervention trial, a technology enhanced randomized trial. Am J Prev Med 2018;55(05): 603-614

25 Peaceman AM, Clifton RG, Phelan S, et al; LIFE-Moms Research Group. Lifestyle interventions limit gestational weight gain in women with overweight or obesity: LIFE-Moms prospective meta-analysis. Obesity (Silver Spring) 2018;26(09):1396-1404

26 Haby K, Berg M, Gyllensten H, Hanas R, Premberg Å. Mighty Mums - a lifestyle intervention at primary care level reduces gestational weight gain in women with obesity. BMC Obes 2018;5(01):16

27 Daly N, Farren M, McKeating A, O'Kelly R, Stapleton M, Turner MJ. A medically supervised pregnancy exercise intervention in obese women: a randomized controlled trial. Obstet Gynecol 2017;130 (05):1001-1010

28 Wang C, Wei Y, Zhang X, et al. A randomized clinical trial of exercise during pregnancy to prevent gestational diabetes mellitus and improve pregnancy outcome in overweight and obese pregnant women. Am J Obstet Gynecol 2017;216(04):340-351

29 Du MC, Ouyang YQ, Nie XF, Huang Y, Redding SR. Effects of physical exercise during pregnancy on maternal and infant outcomes in overweight and obese pregnant women: a meta-analy-

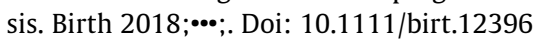

30 Muktabhant B, Ta L, Lumbiganon P, Laopaiboon M. Diet or exercise, or both, for preventing excessive weight gain in pregnancy (review) summary of the findings for the main comparison. Cochrane Database Syst Rev 2015;(06):CD007145

31 Di Carlo C, Iannotti G, Sparice S, et al. The role of a personalized dietary intervention in managing gestational weight gain: a prospective, controlled study in a low-risk antenatal population. Arch Gynecol Obstet 2014;289(04):765-770

32 Horan MK, McGowan CA, Gibney ER, Byrne J, Donnelly JM, McAuliffe FM. Maternal nutrition and glycaemic index during pregnancy impacts on offspring adiposity at 6 months of age analysis from the ROLO randomised controlled trial. Nutrients 2016;8(01):13-15

33 Luo X-D, Dong X, Zhou J. Effects of nutritional management intervention on gestational weight gain and perinatal outcome. Saudi Med J 2014;35(10):1267-1270

34 McGowan CA, Walsh JM, Byrne J, Curran S, McAuliffe FM. The influence of a low glycemic index dietary intervention on maternal dietary intake, glycemic index and gestational weight gain during pregnancy: a randomized controlled trial. Nutr J 2013;12 (01):140

35 Quinlivan JA, Lam LT, Fisher J. A randomised trial of a four-step multidisciplinary approach to the antenatal care of obese pregnant women. Aust N Z J Obstet Gynaecol 2011;51(02):141-146

36 Walsh JM, McAuliffe FM. Impact of maternal nutrition on pregnancy outcome-does it matter what pregnant women eat? Best Pract Res Clin Obstet Gynaecol 2015;29(01):63-78 
37 Althuizen E, van der Wijden CL, van Mechelen W, Seidell JC, van Poppel MNM. The effect of a counselling intervention on weight changes during and after pregnancy: a randomised trial. BJOG 2013;120(01):92-99

38 Asbee SM, Jenkins TR, Butler JR, White J, Elliot M, Rutledge A. Preventing excessive weight gain during pregnancy through dietary and lifestyle counseling: a randomized controlled trial. Obstet Gynecol 2009;113(2 Pt 1):305-312

39 Așcı Ö, Rathfisch G. Effect of lifestyle interventions of pregnant women on their dietary habits, lifestyle behaviors, and weight gain: a randomized controlled trial. J Health Popul Nutr 2016; 35:7

40 Bogaerts AF, Devlieger R, Nuyts E, Witters I, Gyselaers W, Van den Bergh BRH. Effects of lifestyle intervention in obese pregnant women on gestational weight gain and mental health: a randomized controlled trial. Int J Obes 2013;37(06):814-821

41 Huang TT, Yeh CY, Tsai YC. A diet and physical activity intervention for preventing weight retention among Taiwanese childbearing women: a randomised controlled trial. Midwifery 2011; 27(02):257-264

42 Hui A, Back L, Ludwig S, et al. Lifestyle intervention on diet and exercise reduced excessive gestational weight gain in pregnant women under a randomised controlled trial. BJOG 2012;119(01): 70-77

43 Hui AL, Back L, Ludwig S, et al. Effects of lifestyle intervention on dietary intake, physical activity level, and gestational weight gain in pregnant women with different pre-pregnancy body mass index in a randomized control trial. BMC Pregnancy Childbirth 2014;14:331

44 Korpi-Hyövälti EA, Laaksonen DE, Schwab US, et al. Feasibility of a lifestyle intervention in early pregnancy to prevent deterioration of glucose tolerance. BMC Public Health 2011;11:179

45 Luoto R, Kinnunen TI, Aittasalo M, et al. Primary prevention of gestational diabetes mellitus and large-for-gestational-age newborns by lifestyle counseling: a cluster-randomized controlled trial. PLoS Med 2011;8(05):e1001036

46 Mustila T, Raitanen J, Keskinen P, Saari A, Luoto R. Lifestyle counseling during pregnancy and offspring weight development until four years of age: follow-up study of a controlled trial. J Negat Results Biomed 2012;11:11

47 Petrella E, Malavolti M, Bertarini V, et al. Gestational weight gain in overweight and obese women enrolled in a healthy lifestyle and eating habits program. J Matern Fetal Neonatal Med 2014;27 (13):1348-1352

48 Phelan S, Phipps MG, Abrams B, Darroch F, Schaffner A, Wing RR. Randomized trial of a behavioral intervention to prevent excessive gestational weight gain: the Fit for Delivery Study. Am J Clin Nutr 2011;93(04):772-779

49 Poston L, Bell R, Croker H, et al; UPBEAT Trial Consortium. Effect of a behavioural intervention in obese pregnant women (the
UPBEAT study): a multicentre, randomised controlled trial. Lancet Diabetes Endocrinol 2015;3(10):767-777

50 Rauh K, Gabriel E, Kerschbaum E, et al. Safety and efficacy of a lifestyle intervention for pregnant women to prevent excessive maternal weight gain: a cluster-randomized controlled trial. BMC Pregnancy Childbirth 2013;13:151

51 Renault KM, Nørgaard K, Nilas L, et al. The Treatment of Obese Pregnant Women (TOP) study: a randomized controlled trial of the effect of physical activity intervention assessed by pedometer with or without dietary intervention in obese pregnant women. Am J Obstet Gynecol 2014;210(02):134.e1-134.e9

52 Ruchat SM, Davenport MH, Giroux I, et al. Nutrition and exercise reduce excessive weight gain in normal-weight pregnant women. Med Sci Sports Exerc 2012;44(08):1419-1426

53 Szmeja MA, Cramp C, Grivell RM, Deussen AR, Yelland LN, Dodd JM. Use of a DVD to provide dietary and lifestyle information to pregnant women who are overweight or obese: a nested randomised trial. BMC Pregnancy Childbirth 2014;14:409

54 Tanvig M, Vinter CA, Jørgensen JS, et al. Effects of lifestyle intervention in pregnancy and anthropometrics at birth on offspring metabolic profile at 2.8 years: results from the Lifestyle in Pregnancy and Offspring (LiPO) study. J Clin Endocrinol Metab 2015;100(01):175-183

55 Vesco KK, Karanja N, King JC, et al. Efficacy of a group-based dietary intervention for limiting gestational weight gain among obese women: a randomized trial. Obesity (Silver Spring) 2014;22 (09):1989-1996

56 Byrne NM, Groves AM, McIntyre HD, Callaway LK; BAMBINO group. Changes in resting and walking energy expenditure and walking speed during pregnancy in obese women. Am J Clin Nutr 2011;94(03):819-830

57 Garnæs KK, Mørkved S, Salvesen Ø, Moholdt T. Exercise training and weight gain in obese pregnant women: a randomized controlled trial (ETIP trial). PLoS Med 2016;13(07):e1002079

58 Haakstad LA, Bø K. Effect of regular exercise on prevention of excessive weight gain in pregnancy: a randomised controlled trial. Eur J Contracept Reprod Health Care 2011;16(02):116-125

59 Kong KL, Campbell CG, Foster RC, Peterson AD, LanninghamFoster L. A pilot walking program promotes moderate-intensity physical activity during pregnancy. Med Sci Sports Exerc 2014;46 (03):462-471

60 Nascimento SL, Surita FG, Parpinelli MÂ, Siani S, Pinto e Silva JL. The effect of an antenatal physical exercise programme on maternal/perinatal outcomes and quality of life in overweight and obese pregnant women: a randomised clinical trial. BJOG 2011;118(12):1455-1463

61 Ruiz JR, Perales M, Pelaez M, Lopez C, Lucia A, Barakat R. Supervised exercise-based intervention to prevent excessive gestational weight gain: a randomized controlled trial. Mayo Clin Proc 2013;88(12):1388-1397 